\title{
Von der Werkstoffforschung zur Materials Science
}

\author{
Klaus Hentschel
}

From Scattered Research on Diverse Materials to Materials Science

The manipulation of materials, and to some extent also their systematic classification, form an integral part of the skills and culture of all societies. Yet it took long for proper sciences (e.g., metallurgy, glass technology, polymer chemistry or solid-state physics) to develop out of many processing procedures, tapping the accumulated knowledge about specific material characteristics. In the late 20th century an overarching science of workable materials emerged: materials science. This concept and term originated from major boosts in materials research during WWII and the Cold War, first financed by the U.S. Department of Defense and the Advanced Research Project Agency (ARPA). The COSMAT-Report from 1974, written by the American Presidential Science Advisory Committee's "Committee on the Survey of Materials Science and Engineering" four years after its inception, and subsequent reports heralded in the second institutionalization phase of materials science in the USA and with some delay also elsewhere. As the field continued to expand, the demand grew from within in the late 1990s for disciplinary status. This article sorts these claims from the various camps (by solid state physicists vs. chemists vs. engineers) and sets them in the context of unfolding institutional change. The developments within the German-speaking realm, thus far unduly neglected in these debates, are added. I close with a systematic discussion of eight indicator arguments for or against a convergence of this complex field of research into a single coherent discipline. Against Bensaude-Vincent's (2001: 242) thesis that materials science still is an "aggregation of fragments of knowledge", I argue that by 2010 materials science did indeed achieve disciplinary status in a historically rare and enduring process of discipline formation through "emergence by integration" rather than by differentiation.

Keywords: Discipline formation, indicators, materials science and engineering (MSE), metallurgy, metallography, research technologies

Schlüsse/wörter: Entstehung einer wissenschaftlichen Disziplin, Indikatoren, C.S. Smith, R.W. Cahn, B. BensaudeVincent

Mit dem vorliegenden ersten Beitrag zum Themenheft zur Geschichte der Materialforschung wird ein doppeltes Anliegen verfolgt: einerseits eine breit angelegte Literaturübersicht zum Thema zu bieten und andererseits den Wandel dieses Forschungsfeldes und seine zunehmende Institutionalisierung im 20. Jahrhundert darzustellen. Pionierbeiträge leisteten dabei unter anderem 
die beiden Metallurgen Cyril Stanley Smith (1903-1992) und Robert W. Cahn (1924-2007). ${ }^{1}$ Als größte Kernfächer, aus denen wichtige Zweige der Materialforschung erwuchsen standen dabei im Zentrum: theoretische Konzepte, Experimentalverfahren und instrumentelle Analysetechnik der Metallurgie sowie Forschungstechnologien der Festkörperphysik beziehungsweise der physikalischen Chemie.

Die disziplinären Zuordnungen, die um 1900 noch völlig unstrittig und unproblematisch waren, sind angesichts des hybriden Status der Materialwissenschaften in heutigen Universitäten, Technischen Hochschulen, außeruniversitären Forschungsinstitutionen und industriellen Laboratorien zunehmend schwieriger geworden. Es fällt auf, dass sich seit $1975 \mathrm{immer}$ mehr werkstoffkundlich arbeitende Laboratorien und Studiengänge zu einer Umbenennung entschlossen haben, die deren Wurzeln in Chemie, technischer Werkstoffkunde, Metallurgie, Physik, um nur ein paar zu nennen, zugunsten einer einheitlichen Designation als materials science and engineering zumindest überdecken. Ist materials science mittlerweile zu einer eigenen Disziplin geworden, vielleicht sogar einer Art „Superdisziplin“, wie einige ihrer Akteure zu glauben scheinen? Oder ist ihr Aufkommen (ebenso wie in jüngster Zeit die Nanotechnologie, siehe Schummer 2011) umgekehrt ein Indiz für die zunehmende Auflösung disziplinärer Kohärenz zugunsten interdisziplinärer, aber zugleich lockerer und fließender Forschungsverbünde? Selbst unter den materials scientists ist dieser Punkt bis heute umstritten - so plädierte Martin Stratmann, Direktor des Max-Planck-Instituts für Eisenforschung, 2006 eher für die Deutung als eine Art Querschnittsfeld, während der britische Metallkundler Robert W. Cahn (1924-2007) die Auffassung vertrat, die Materialwissenschaft sei seit den 1990er Jahren dabei, sich durch zunehmende Kohärenz der Forschungsmethoden aus einem eher losen Verbund zu einer eigenen, konvergenten Disziplin zu entwickeln. Bernadette Bensaude-Vincent kam hingegen 2001 in ihrer wissenschaftshistorischen Betrachtung zu dem Schluss, dass eine solch disziplinäre Ausreifung des Forschungsfeldes nicht eingetreten sei, das sich ihr eher als ein loses Agglomerat darstellte.

Im vorliegenden Beitrag werden die Positionen einflussreicher Akteure als Funktion der Zeit gegenübergestellt und vor dem Hintergrund ihrer unterschiedlichen disziplinären Herkünfte, Interessen und Problemlagen eingeordnet. $\mathrm{Zu}$ deren Bewertung werden die aus der Wissenschaftsgeschichte, -soziologie und -theorie bekannten Modelle und Kriterien für Disziplinengenese zu Rate gezogen. Als Grundlage zur Systematisierung des Disziplinbegriffs und Prüfung der Ansprüche auf Disziplinstatus der materials science dienen mir acht strukturelle und allgemein akzeptierte spezifische Indikatoren ${ }^{2}$ : kohärentes Set von Paradigmen und Methoden, Lehrbücher, universitäre Lehreinheiten und Studiengänge, Lehrstühle und Institute, Zeitschriften, fachspezifische wissenschaftliche Vereinigungen, professionelle und persönliche Interaktionen und eine Gruppenidentität als Ab- und Ausgrenzungsmerkmal. 
Nach einer weitgehend chronologischen Darlegung der Traditionen von Materialforschung werden die Phasen ihrer Institutionalisierung aufgerollt. Dafür kennzeichnend sind Materialprüfungsanstalten, industrielle Materialforschungslaboratorien und universitäre interdisciplinary laboratories in den USA mit spezifischer Ausrichtung auf Werkstoffforschung gewesen. Ein weiterer Meilenstein in der Problembündelung, aber auch in der öffentlichkeitswirksamen Werbung für die Anliegen und Methoden der Materialforschung war der COSMAT-Report der US-amerikanischen National Academy of Sciences von 1974 sowie dessen Nachfolgeberichte. Abschließend wird der gegenwärtige Zustand des komplexen Forschungsfeldes vor dem Hintergrund der obigen Indikatoren geprüft und in Ergänzung zu Bensaude-Vincent die These vertreten, dass sich die materials science dann seit 2000 in der Tat zu einer veritablen Disziplin entwickelt hat. Insbesondere ist nun auch die Einigung auf generische (materials catholic) Deskriptoren erfolgt, die zur Charakterisierung vieler Stoffklassen geeignet sind. ${ }^{3}$ Damit ist die praktisch ein ganzes Jahrhundert lang andauernde Formierungsphase jenes ungewöhnlichen Disziplinbildungsprozesses als einer „emergence by integration “4 vorläufig abgeschlossen.

\section{Vom handwerklichen Können zu verschiedenen werkstoffbezogenen Wissenschaften}

Lange Zeit blieben Kenntnisse über Materialien Praktikerwissen. Ihre Tradierung erfolgte meist in mündlicher Form durch Weitergabe von Rezepten vom Meister zum Schüler. Oft handelte es sich bewusst um geheim gehaltenes Wissen, so etwa bei den Verfahren zur Verhüttung von Eisenerzen, die im Hethitischen Großreich spätestens seit dem 17. Jahrhundert vor Christus belegt sind, sich aber erst nach dem Niedergang desselben im frühen 12 . Jahrhundert nach Christus allmählich über den vorderen Orient auf den Mittelmeerraum ausbreiteten (Raymond 1986, Kap. 3, Hummel [1998] 2004, Kap. 7). Seit dem ausgehenden Mittelalter gibt es vermehrt Traktate von Praktikern (wie etwa Probier-, Bergwerks- und Steinmetzbüchlein, Maschinenbücher und Büchsenmeistertraktate), in denen durchaus einschlägiges Material- und Verfahrenswissen festgehalten wurde, wenn auch häufig in zumindest für uns heute - schwer reproduzierbarer Form (Kranzberg/Smith 1979: 16 f.). Aus dem Lehrkanon dieser Zeit wurde jenes Wissen jedoch noch weithin ausgegrenzt. In der frühen Neuzeit fand dann ein langsames Aufnehmen dieser praktischer Wissensstränge auch durch systematisch und auf Theorie abzielende Gelehrte statt: Agricola beobachtete die in den Bergwerken eingesetzte Hebetechnologie und beschrieb sie 1556 anschaulich in De re metallica und Galilei ließ sich in den Arsenalen Venedigs zu seiner neuen 
Wissenschaft von der Festigkeit der Materialien inspirieren, wie er selbst 1638 zu Beginn seiner Discorsi schrieb. Otto von Guericke (1602-1686) arbeitete schließlich zusammen mit Pumpenmachern und Kupferhauern, die ihm seine Vakuumgefäße herstellten und abdichten halfen.

Neben der Festigkeitsmechanik und Elastizitätslehre (Leon 1912, Timoshenko 1953, Kahlow 1991, Johnson 2009 entstanden weitere frühe Zonen systematischer Materialforschung in der (Al-)Chemie, der Metallurgie sowie in den Spezialforschungen zur Glas- und Porzellanherstellung, so etwa die Glasmacher-Traktate von Johannes Kunckel (um 1630-1703). Der Kurator der Royal Society, Robert Hooke (1635-1703), betrachtete um 1660 verschiedenste Materialien unter dem Mikroskop und setzte ihre Festigkeitsund Torsions-Eigenschaften mit ihren Strukturen in Beziehung. Seine Micrographia aus dem Jahre 1665 wurde vereinzelt wegen des Einsatzes von Mikroskopie sogar als eine Vorstufe der späteren Metallographie gewertet (Smith 1969, Mehl 1948: 12). Der „physical metallurgist“ Cahn (1995: 1506 f.) hingegen interpretierte diese frühen Mikrostrukturuntersuchungen als eine subscidiary science, wobei er den Neologismus subscidy als „subsidiary scientific domain", also unterhalb der Disziplinenebene liegend, definierte. Réaumur versuchte dann im 18. Jahrhundert derartige Erkenntnisse angereichert um chemische Einsichten über den Einfluss von Salzen, Schwefel und Kohlenstoff, gezielt zur Herstellung verbesserter Stähle zu nutzen. ${ }^{5}$ Michael Faraday (1791-1867) arbeitete an der Royal Institution jahrelang an der Analyse und Optimierung von Stählen und hochbrechendem Bleiglas. ${ }^{6}$ Diese Beispiele repräsentieren zwar bereits systematisierte Formen der Materialbeziehungsweise Werkstoffforschung, aber sie bleiben weit voneinander getrennte, hoch spezialisierte Untersuchungsbereiche, praktiziert von völlig verschiedenen Trägergruppen wie etwa „Maschinenkünstlern“ und „Mühlenärzten“, Künstler-Ingenieuren, Bergassessoren oder gentleman scientists. Es handelte sich dabei also keineswegs um eine Vorform von Werkstoffwissenschaft beziehungsweise materials science (jeweils im Singular). Ihre Ursprünge liegen an der Wende zum 20. Jahrhunderts, wofür im folgenden zwei Gründe ausgeführt werden sollen: die Interdependenz von Materialforschung und research technologies und die durch die beiden Weltkriege ausgelöste Explosion der Materialvielfalt.

\section{Die Interdependenz von Materialforschung und research technologies}

Der wissenschaftliche Zugang zu Materialien unterscheidet sich vom praktisch-handwerklichen unter anderem in einer stärkeren Berücksichtigung von 
Prüf- und Messverfahren. Deshalb war die Herausbildung der Werkstoffwissenschaft beziehungsweise der materials science auf das Engste mit der Geschichte von Forschungstechnologien (research technologies) verknüpft, für die Terry Shinn folgende drei Strukturmerkmale aufgezeigt hat (Shinn/Joerges 2001): erstens eine generische Anwendbarkeit in den verschiedensten Disziplinen, zweitens die „Interstitialität“ der Akteure, die zwischen verschiedenen Disziplinen hin- und herwechseln, oft auch zwischen Universität, Industrie und außeruniversitären Forschungsinstitutionen und drittens die Ausprägung einer jeweils eigenen Metrologie. ${ }^{7}$

Die meisten Forschungstechnologien des 20. Jahrhunderts hatten zum Teil erheblichen Einfluss auf die Werkstoffprüfung. So wurde etwa mit den 1896 entdeckten Röntgenstrahlen ein zerstörungsfreier Blick in das Innere von partiell strahlungsdurchlässigen Materialien möglich. Die Röntgenbeugung ( $x$-ray diffraction, vgl. Bragg 1945, Ewald 1962, Eckert 1993: 45-48, Lemmerich 1996) ermöglicht die Strukturaufklärung von Kristallen durch Interferenzmuster, die durch Reflektion an periodischen Gitterebenen erzeugt wird. Mithilfe der Transmissionselektronenmikroskopie können hauchdünn geschliffene Materialproben auf feinste Mikrorisse oder andere Inhomogenitäten untersucht werden, ${ }^{8}$ während die Raster-Tunnelmikroskopie Strukturaufklärungen und zum Teil auch Veränderungen der Materie auf atomarer Skala ermöglicht. ${ }^{9}$

Einige wenige dieser Forschungstechnologien setzten bereits im 19. Jahrhundert ein, so etwa die chemische Spektralanalyse um 1860 (Hentschel 2002) oder elektrische Widerstandsmessungen 1827 mit der Klärung des zugrundeliegenden Konzepts durch Georg Simon Ohm (1789-1854). Sie waren von großer praktischer Relevanz zum Beispiel bei der Prüfung der elektrischen Leitfähigkeit verschiedenster Legierungen für die transatlantischen Unterwasserkabel ab den 1850ern, für die neben den Kupferlegierungen auch das isolierende Naturgummi (gutta percha) entscheidend war (Smith 1977: 22-28, Hunt 1997). Zum Einsatz von Forschungstechnologien für die Zwecke der Materialforschung auf breiter Front kam es jedoch erst nach der Entdeckung der Röntgenstrahlen, des Elektrons sowie weiterer mit Radioaktivität verbundener Strahlungsformen. Strukturaufklärungen, die erlaubten in die atomare beziehungsweise molekulare Ebene der untersuchten Materialien vorzudringen, wurden ermöglicht. Mit der Entwicklung kern- und festkörperphysikalischer Methoden intensivierten sich diese Untersuchungsmöglichkeiten. Viele dieser Forschungstechnologien, darunter zum Beispiel die kernmagnetische Resonanz, stammten zwar aus der Physik, wurden aber bald darüber hinausgehend auch in ganz anderen Gebieten eingesetzt, so etwa auch in der Medizin, womit sich das erste der drei Kriterien von Shinn, das der Generizität, erfüllte. ${ }^{10}$ Auch Cahn (1995: 1550) sieht in der Bereitstellung von Untersuchungsmethoden den wichtigsten Beitrag der Physik zur ansonsten eher von der Chemie und Technik her bestimmten Materialwissenschaft. 
Eine andere Technik, die sogenannte Metallographie, in der feinste Struktureigenschaften von Proben durch Anschliff und chemische Ätzung mit nachfolgender Betrachtung und gegebenenfalls photographiert oder unter dem Mikroskop sichtbar gemacht werden konnten, kam aus der Mineralogie und Kristallographie. Der Pionier dieses Verfahrens war Henry Clifton Sorby (1826-1908) aus Sheffield. ${ }^{11}$ Sorby hatte sich durch die Widmanstätten'sche Präparation von Meteoriten zu einem analogen Verfahren mikroskopischer Untersuchung von angeschliffenen und glatt polierten Metalloberflächen anregen lassen. In Deutschland entwickelten der Eisenbahningenieur und Professor an der Technischen Hochschule Berlin-Charlottenburg Adolf Martens (1850-1914), der auch das 1904 gegründete Materialprüfungsamt in Berlin-Dahlem leitete, sowie sein Mitarbeiter, der Eisenhüttenkundler Emil Heyn (1867-1927), diese Methoden weiter. ${ }^{12}$ Seit dem frühen 20. Jahrhundert wurde die Metallographie in großem Maßstab für Untersuchungen von Mikrostrukturen eingesetzt, zum Beispiel um auf diese Weise Risse und Bruchflächen, Korn- und Phasengrenzen, durch Druck, Torsion, Temperaturschwankungen oder andere äußere Einflüsse im Material entstandene Deformationen, Oxidationen und Erosionen in Materialproben zu untersuchen. $^{13}$ In großangelegten Tafelwerken mit Hunderten solcher Metallographien erlernten die Praktiker, wie die Dünn- und Anschliffe der verschiedensten metallischen Werkstoffe im Normalfall auszusehen hatten und woran man auch die kleinsten Anzeichen von Schwachstellen ausmachen konnte. ${ }^{14}$

Die Industrialisierung sowie der intensive Ausbau des Eisenbahnwesens seit der Mitte des 19. Jahrhunderts führten zu ständig steigenden Anforderungen an die Festigkeitseigenschaften der metallischen Werkstoffe, insbesondere der Stähle, die geprüft, klassifiziert und taxiert werden mussten (Ude 1935). Für die Krupp'schen Stahlwerke wurden die ersten Materialprüfungen in den 1860er Jahren in der Privatanstalt von David Kirkaldy (18201897) in London vorgenommen, wo Krupp auch seine ersten Prüfgeräte her bezog. Auf Anregung einer technischen Kommission des Vereins deutscher Eisenbahnverwaltungen erschienen 1877 beziehungsweise 1879 Denkschriften, in denen erstmalig Mindestwerte für Zugfestigkeits- und Brucheinschnürwerte festgelegt beziehungsweise die Gründung staatlicher Prüfanstalten für diese Untersuchungen gefordert wurden. ${ }^{15}$ Eine weitere Vereinheitlichung der Prüfverfahren und -geräte wurde auf den ersten Konferenzen zur Vereinbarung einheitlicher Prüfungsmethoden in München 1884 beziehungsweise in Dresden 1886 beschlossen, aus denen 1896 auch der deutsche Verband für die Materialprüfungen der Technik hervorging. ${ }^{16}$ Neue visuelle Methoden der Materialanalyse wurden entwickelt. In der Abbildung 1 ist rechts die durch das Glühen im Material induzierte Verkleinerung der Korngrenzen deutlich erkennbar. Unten rechts ist dabei die gewählte mikroskopische Vergrößerung angegeben. In Abbildung 2 ist die stark von der Seite kommende Beleuchtung 


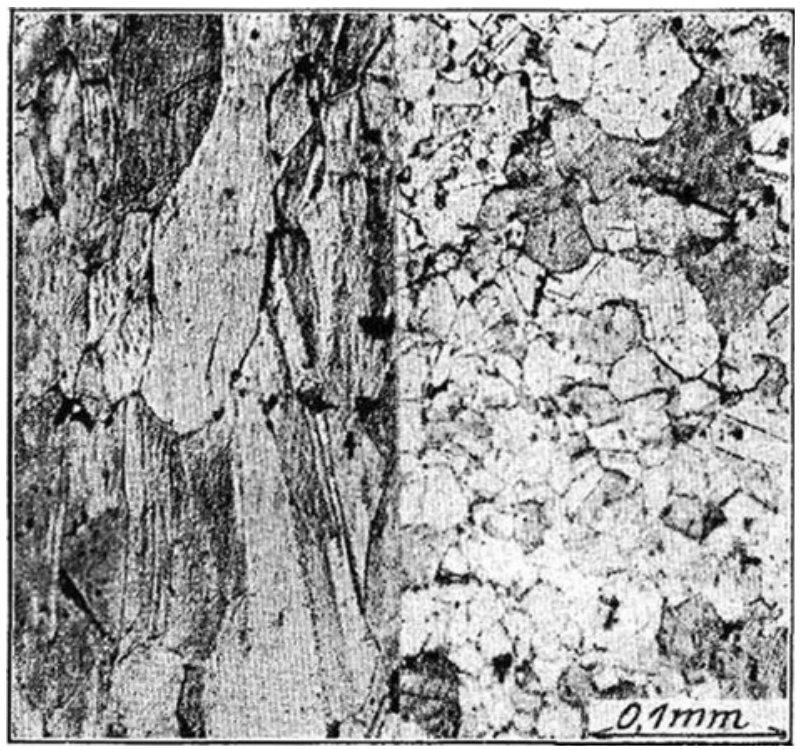

Abb. 1 Ein metallographischer Vergleich von kaltgezogenem Messing (links) mit geglühtem Messing (rechts) (Baumann 1920: Abb. 28)

der Probe zu sehen. Sie brachte zwar gute Kontraste und Schattierung von Vertiefungen, aber nur geringe Tiefenschärfe mit sich, weshalb Martens (übrigens ebenso wie Sorby) die meisten seiner metallographisch untersuchten Proben noch zeichnete und nicht photographierte. So betonte er, dass kein Mittel so sehr die Schärfe der Beobachtung übt wie das Nachzeichnen des im Mikroskop gesehenen. (Martens 1880).

Die Metallkunde als Disziplin wandelte sich weitgehend durch dieses Eindringen neuer, visueller Forschungstechnologien der Metallographie. In den 1870ern begann auch die Geschichte der eigens zum Zweck der Materialprüfung gegründeten Institute, wie etwa dem Münchener Mechanisch-Technischen Laboratorium, der Eidgenössischen Materialprüfungs- und Forschungsanstalt (gegr. 1879) sowie analoge, heute noch existierende Materialprüfungsanstalten in Stuttgart oder Darmstadt (abgekürzt MPAs, gegründet 1884 beziehungsweise 1907), mit amtlichem Prüfsiegel, für staatliche und private Auftraggeber. ${ }^{17}$ Die dort praktizierten Mess- und Prüfverfahren wurden - nicht zuletzt aufgrund der gebotenen Gleichbehandlung aller Auftraggeber - weiter standardisiert und verfeinert und fanden ihren Niederschlag in entsprechenden Messgeräten, Lehrund Handbüchern, an deren diversen Auflagen der Fortschritt durch weitere angewandte Forschungstechnologien ablesbar ist. ${ }^{18}$ Frühe Pioniere der Werkstoffprüfung wie etwa Franz Carl Achard (1753-1821), August Wöhler (18191914) oder Johann Bauschinger (1834-1893) arbeiteten als Physiker, Chemiker oder Ingenieure an Polytechnischen Hochschulen oder Materialprüfanstalten. ${ }^{19}$ Der erste deutsche Lehrstuhl für Werkstoffkunde wurde 1927 an der 


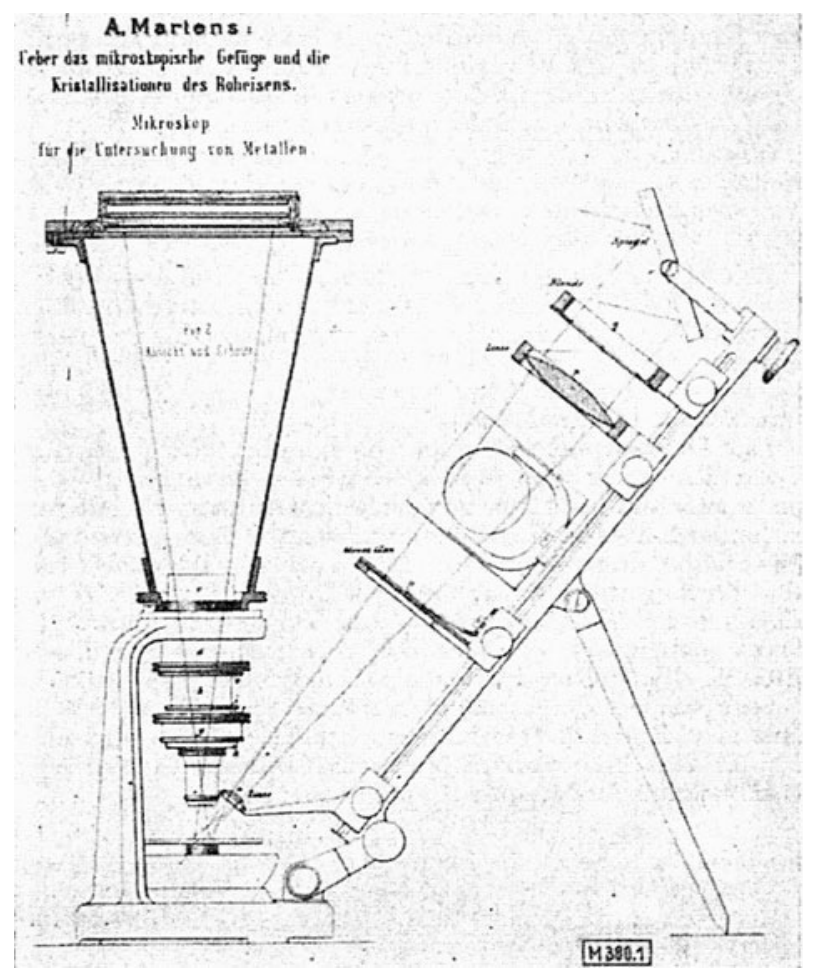

Abb. 2 Querschnitt-Zeichnung zum Aufbau des ersten Entwurfs eines Metall-Mikroskops (Martens 1880: Tafel XXI)

Großherzoglichen Technischen Hochschule Darmstadt eingerichtet; weitere folgten unter anderem in München, Berlin (1935), Hannover (1935), Braunschweig und Stuttgart, oft in Betriebseinheit und zum Teil auch Personalunion mit den Besetzungen der Leitungspositionen in den jeweiligen regionalen MPAs. Parallel dazu entstanden auch in der Großindustrie einerseits eigene Materialprüfungseinrichtungen zur Qualitätssicherung der Produktion und eingehender Lieferungen, andererseits gut ausgestattete Forschungslaboratorien mit spezieller Ausrichtung auf Materialfragen. ${ }^{20}$

\section{Materialforschung im Zweiten Weltkrieg und im Kalten Krieg}

Die Materialengpässe im Ersten und Zweiten Weltkrieg führten jeweils zur Suche nach neuen Materialien: entweder nach besseren als den altbekannten oder nach notdürftigen Ersatzmaterialien aufgrund von Mangelerscheinungen infolge der Kriegsblockaden, insbesondere künstliches Gummi, Treibstoffe und Öle sowie Leichtmetalle beziehungsweise Ersatzstoffe. Während diese 
Materialengpässe im Ersten Weltkrieg oft zu spät bemerkt wurden, um noch wirksame Gegenmaßnahmen einzuleiten, bemühte man sich im Zweiten Weltkrieg auf beiden Seiten der Front um die Behebung oder Kompensation der knappen Ressourcen. Gründungen wie die des NS-Reichsamtes für Wirtschaftsausbau, die Schaffung eines Reichsforschungsrates beziehungsweise eines National Research Council in den USA sollten Abhilfe schaffen. Trotz polykratischer Machtstrukturen im Nationalsozialismus wurden in vielen Sektoren Engpässe durch die Entwicklung beziehungsweise industrielle Bereitstellung von Ersatzstoffen wie etwa Aluminium-Zink-MagnesiumLegierungen als „Deutsche Metalle“ abgemildert (Maier 2002, 2006, Luxbacher 2010, 2011). Ferner wurden durch technische Vereine und Institutionen wichtige Normen festgelegt und Beschlüsse gefasst (dazu Maier, in Vorbereitung). Auf jeden Fall wurden zentralisierte Strukturen geschaffen, die erst in der nachfolgenden Phase des Kalten Krieges ihre volle Wirksamkeit entfalteten.

In den 1950er Jahren empfahl das Science Advisory Committee des USPräsidenten "materials as a priority“ in Forschung und Entwicklung. ${ }^{21}$ Der Sputnik-Schock von 1957 verstärkte die Bemühungen um bemannte Raumfahrt mit ihren extremen Materialanforderungen. Insbesondere das Verteidigungsministerium der Vereinigten Staaten finanzierte viele Spezialuntersuchungen und -entwicklungen für Waffen-, Marine- sowie Luft- und Raumfahrtprojekte. Die Advanced Research Project Agency (ARPA) schloss Verträge mit etlichen Universitäten, zum Beispiel 1961 mit Harvard, dem MIT, Brown, Stanford und Chicago für 13 Millionen US-Dollar. ${ }^{22} 1957$ gingen 35 Prozent der investierten Gelder in die Festkörperphysik, 29 Prozent in die Metallurgie, zehn Prozent in die Erforschung von Keramiken. Ingesamt zwölf sogenannte Interdisciplinary Materials Laboratories wurden zwischen 1960 und 1962 von ARPA nach dem Vorbild bereits gut funktionierender, ebenso interdisziplinärer Radar-, Elektronik- und Kernforschungslaboratorien gegründet, die neben den großen Industrieforschungslaboratorien zu einem weiteren Nukleus für die sich institutionell konsolidierende Materialforschung wurden (Bensaude-Vincent 2001).

\section{Von der Materialforschung zur materials science}

In diesem Kontext wurde ab den 1950er Jahren auch der Ausdruck material(s) science(s) von einigen maßgeblichen Akteuren benutzt, oft unter dem Zusatz materials science and engineering (MSE). Mit der Umbenennung etlicher Interdisciplinary Materials Laboratories in Material Research Labs (MRL), so etwa an den Universitäten Cornell, Pennsylvania, Northwestern, sowie durch analoge Umtaufungen ganzer Fachbereiche an US-Universitäten wurden diese Institutionalisierungsschritte um 1970 fortgesetzt. $^{23}$ Hierzu 
einige Graphiken: In Abbildung 3 ist die durch die ARPA-Unterstützung ausgelöste erste Gründungswelle ab 1960 sowie der Zuwachs an Umbenennungen in MSE nach 1981 zu sehen, die auf eine Initiative der National Science Foundation zur Förderung von „University Industry Cooperative Research Centers" zurückgeht (Groenewegen/Peters 2002: 121-123). In Abbildung 4 ist die Stagnation in Bereichen wie Keramik- oder Polymerforschung sowie die stetige Abnahme in Metallurgie zu sehen: Während Festkörperphysik 1970 bis 1980 sinkt und dann bis 1990 stagniert, ist MSE das einzige fast kontinuierlich steigende Fach.

Wie noch gezeigt werden wird, traf in Europa und andernorts die Welle von Umbenennungen von Gesellschaften, Instituten und Laboratorien mindestens erst ein Jahrzehnt später ein. Eine analoge Tendenz gab es auch in der Industrie, so zum Beispiel in den Bell Telephone Laboratories in New Jersey, wo 1947 der Transistor entwickelt worden war (Hoddeson 1977), und im

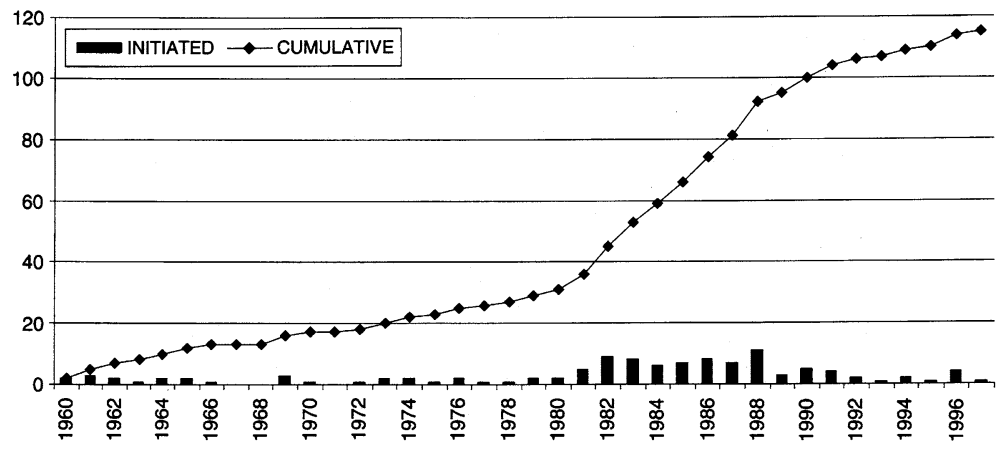

Abb. 3 US Forschungszentren für MSE 1960-1997 (Groenewegen/Peters 2002: 129)

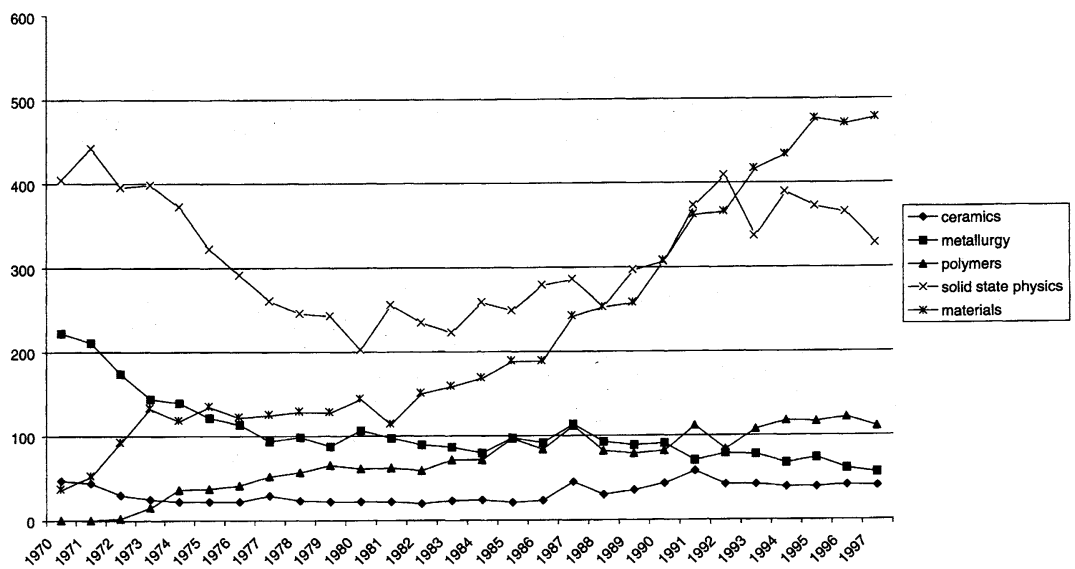

Abb. 4 Promotionsabschlüsse an US-amerikanischen Universitäten 1970-1998 aufgegliedert nach disziplinären Feldern (Groenewegen/Peters 2002: 130) 
General Electric Corporate Laboratory in Schenectady. Bis 1987 existierten bereits 17 staatliche MRLs in den USA. Differenziert man deren Personal nach akademischen Abschlüssen in den Mutterdisziplinen auf, so ergeben sich in etwa folgende Verteilungen: 35 Prozent Physiker, 25 Prozent Chemiker, 19 Prozent Metallurgen, 16 Prozent andere Ingenieurwissenschaften, fünf Prozent andere Ausbildungsfächer der in MRL angestellten Materialforscher. ${ }^{24}$ Auch das Energieministerium der Vereinigten Staaten finanzierte MRLs zum Beispiel Neutronenstreuungs- oder Materialermüdungsuntersuchungen, Reaktorenprogramme und Synchrotron-Strahlungsexperimente an Beschleunigern in Stanford oder Brookhaven.

1958/59 wurde das erste Department of Metallurgy an der Northwestern University in Illinois in Graduate Department of Materials Science umbenannt. Analog folgten unter anderem Texas und das MIT, so 1967 in Metallurgy and Materials Science und 1974 in Materials Science and Engineering. Bis 1969 beschlossen immerhin circa 30 Prozent aller US-Departments of Metallurgy den Begriff materials beziehungsweise materials science mitaufzunehmen. Während es inzwischen in den angelsächsischen und deutschsprachigen Ländern kaum noch echte Departments of Metallurgy gibt, besteht in Frankreich die traditionell starke Kopplung von Metallurgie und Chemie bis heute. An der Northwestern University wurde 1959 folgende Begründung für die Umbenennung gegeben:

Traditionally, the field of material[s] science has developed along somewhat
separate channels - solid state physics, metallurgy, polymer chemistry, inorganic
chemistry, mineralogy, glass and ceramic technology. Advance in materials science
and technology is hampered by this artificial division of the whole science into
separate parts. [...] advantages of bringing together a group of specialists in the
various types of materials and allowing and encouraging their cooperation and free
interchange of ideas. (Fellman 2005)

Weitere Gründe für diesen lange noch heftig umstrittenen Namenswechsel waren ein intensiviertes Interesse an Mikrostruktur als prüfbares Indiz für Eigenschaften, gekoppelt an den Import von Analysetechniken der Festkörperphysik und festkörpertheoretische Konzepte wie Gitter und Fehlstellen. ${ }^{26}$ Durch diese neuen Möglichkeiten der wissenschaftlichen Strukturaufklärung änderte sich auch das Selbstverständnis der Metallurgen. Vormals stark auf die Verbesserung und Kontrolle der industriellen Produktion metallischer Werkstoffe ausgerichtet, verschob es sich nun in Richtung Strukturaufklärung, Verstehen von Festkörpereigenschaften und teilweise auch deren gezieltes Beeinflussen (Design-Materialien), ${ }^{27}$ was in der Chemie und Legierungskunde bereits seit dem ausgehenden 19. Jahrhundert aufgekommen war. Daraus resultierte eine „transformation of metallurgy from an empirical art to a field of study based on principles of physics and chemistry" oder wie der Manager des Forschungslaboratoriums für Metalle und Keramiken der General Electric, J. Herbert Hollomon (1919-1985) es 1958 in Form einer chemischen 
Umwandlung formulierte: „out of metallurgy, by physics, comes materials science. ${ }^{\text {“28 }}$ Während er dort der metals and ceramics group vorstand, leitete der Festkörperphysiker William B. Shockley (1910-1989) in den Bell Laboratories die ebenso interdisziplinär besetzte Halbleitergruppe, der 1947 die Entwicklung des Bipolartransistors gelang, für die er zusammen mit John Bardeen and Walter Houser Brattain 1956 den Nobelpreis für Physik erhielt (Queisser 1985: 104, Hoddeson 1977, Hoddeson/Braun/Teichmann/Weart 1992: 406-482, Riordan/Hoddeson 1997). Gerade in industriellen Laboratorien funktionierte die hybride Mischung von Natur- und Technikwissenschaften im Dienste der Materialforschung bereits ausgezeichnet, lange bevor es dafür einen neuen Namen gab.

\section{Der COSMAT-Report von 1974}

Eine zweite, intensivierte Institutionalisierungsphase erfolgte in den 1970er Jahren, für die 1969 das National Colloquy on the Field of Materials (Roy 1970) den Auftakt bot, aus dem 1970 das Committee on the Survey of Materials Science and Engineering (COSMAT) entstand. Bemerkenswert daran ist übrigens, dass neben Naturwissenschaftlern und Ingenieuren auch der Metallurge Cyril S. Smith (1903-1992) und der Technikhistoriker Melvin Kranzberg (1917-1995) an diesem Komitee beteiligt waren, in das somit ausdrücklich historische Perspektiven Eingang fanden. 1974 erschien der entsprechende Bericht der National Academy of Sciences unter dem Titel: Materials and Man's Needs. Materials Science and Engineering COSMAT 1974), ein Startsignal für analoge Bestrebungen auch in anderen Ländern. Dass diese zweite Institutionalisierungsphase der MSE gerade zu diesem Zeitpunkt stattfand, ist auf eine ganze Reihe von Gründen zurückzuführen: die Ölkrise 1973, zunehmende Umweltprobleme, die Gründung des White House Energy Policy Office, das Gesetz zur Luftreinhaltung (Clean Air Act), ab 1970 eine forcierte Katalysatorforschung sowie die 1972 erfolgte Gründung des Umweltprogramms der Vereinten Nationen, intensivierte Materialforschung in Spezialgebieten (so für Kernreaktoren, Automobilindustrie, Luft- und Raumfahrt) sowie Halbleiter für Mikroelektronik. Neu war damals der Einbezug von Materialzyklen und Abfallprodukten, veranlasst durch die rasch wachsenden Müllhalden der Konsumgesellschaft.

Im COSMAT-Report von 1974 wurde geschätzt, dass circa 500.000 Naturwissenschaftler und Ingenieure über Themen der Materialforschung arbeiten, von denen damals maximal 50.000 akademische Abschlüsse hatten, in denen das Wort ,Material' explizit vorkam - alle anderen hatten traditionelle Abschlüsse in Chemie, Physik oder diversen Ingenieurwissenschaften wie 
etwa Metallurgie oder Verfahrenstechnik. 1974 gab es 35 nationale Gesellschaften für beispielsweise Werkstoffwissenschaft, Materialwissenschaft und materials science, ferner im angelsächsischen Bereich an die 90 materialsdesignated BA-Studiengänge sowie circa 50 Graduierten-Studiengänge, die den Begriff ,Material' explizit führten. Der Terminus ,Materialwissenschaft' wurde in jenem Bericht wie folgt definiert: „Materials science and engineering is concerned with the generation and application of knowledge relating the composition, structure, and processing of materials to their properties and uses." (COSMAT 1974, 1).

Die näheren Erläuterungen umfassten ein breites Aufgabenfeld, das von chemischer Analyse und Strukturaufklärung bis zur Herstellung und Laborsynthese sowie zur Verarbeitung in großindustriellem Maßstab reichte und insofern klassische Aufgaben der Chemie, Physik sowie des Anlagenbaus und der Verfahrenstechnik einbezog, und zumindest auch teilweise Umweltverträglichkeitsprüfungen. 1973, ein Jahr zuvor, war an der Penn State University die Materials Research Society gegründet worden. Dabei war bewusst auf die enge quasi-disziplinäre Bezeichnung materials science zugunsten des breiteren materials research verzichtet worden. Im Programm der Universität wurde sie als ,interdisciplinary goal-oriented basic research on materials of technological importance ${ }^{\text {“29 }}$ breit ausgelegt. Eine Definition mit der sich niemand, der in welcher Disziplin auch immer über Materialien forschte, mehr ausgegrenzt fühlen musste.

Im deutschen war der Ausdruck, Materialwissenschaft' damals noch nicht gebräuchlich. Wenn überhaupt, sprach man in den 1970er Jahren eher noch von ,Werkstoffkunde' oder ,Werkstoffwissenschaft', so etwa in dem 1972 in erster Auflage und bis 2003 in neun weiteren Auflagen erschienenen Lehrbuch von Werner Schatt ("1923) aus Dresden oder in den zeitgleich verfassten hochschuldidaktischen Überlegungen von Klaus Urban (1972). ${ }^{30}$ Interessanterweise scheint der letztgenannte Terminus eine Ost-West-Wanderung durchlaufen zu haben. Anfangs fast nur in der Deutschen Demokratischen Republik verwendet, dann zögerlich und in scare quotes von Köster (1969: 666) übernommen, wurde er später für einschlägige Schriftenreihen von westdeutschen Verlagen (wie etwa des Aachener Shaker-Verlags ab 1999) benutzt und ist mittlerweile praktisch synonym mit ,Materialwissenschaft'. Die neutraleren, viel älteren Termini wie ,Werkstoffkunde' oder,Werkstoffprüfung' und andere Komposita wurden jedoch nach dem Zweiten Weltkrieg in beiden Hälften Deutschlands gleichermaßen verwendet und sind bis heute verbindlicher Bestandteil der Grundausbildung aller Ingenieurwissenschaften. Trotz der gebräuchlicher werdenden Verwendung von materials science/s (noch häufig im Plural) beziehungsweise ,Werkstoffwissenschaft' (meist bereits im Singular) waren sich die Akteure in Ost und West um 1974 doch weitgehend darüber einig, dass noch keine Konvergenz zu einer neuen Disziplin vorlag: 
Materials science and engineering is not a single discipline, like physics or chemistry, but rather a multi-disciplinary area of research and engineering that has evolved gradually over the last twenty years into a coherent aggregation of related activities, but within each of the component disciplines retains its identity. ${ }^{31}$

Im Nachfolgereport von 1975 einigte sich das COSMAT Komitee auf die griffige Formel von „materials science and engineering [MSE] as a multidiscipline" (COSMAT 1975: Kap. 3, Kranzberg/Smith 1979: 28). Unterstützt wurde diese Rhetorik von einer eingängigen Graphik, die das große Feld wie eine Torte in handliche disziplinär verortete Stücke aufteilte, mit einem Kernbereich von Physik, Chemie und Mathematik und radial nach außen folgenden Zonen zunehmender Anwendungsbezogenheit (vgl. Abb. 5). ${ }^{32}$ Irreführend daran war unter anderem, dass in jener „multi-discipline“ MSE de facto kein gerichteter Gradient von Wissenschaft zur Technik bestand, sondern beide in Lehre und Forschung in Hybriden ohne hierarchische Überoder Unterordnung miteinander arbeiteten.

In Konkurrenz dazu stand ein noch stärker aus physikalischer Perspektive gewonnenes Diagramm (vgl. Abb. 6), in dem die Festkörperphysik alleine im Zentrum dargestellt wurde, umringt von der materials science, um das sich alle anderen Fachsparten sich paarweise überlappend wie Blütenblätter herumgruppierten. Für manche Physiker stellte sich die Materialwissenschaft als eine Art ausgebauter Festkörperphysik dar, ${ }^{33}$ und damit als eine Kombination aus beispielsweise angewandter Quantenmechanik und Quantenchemie,

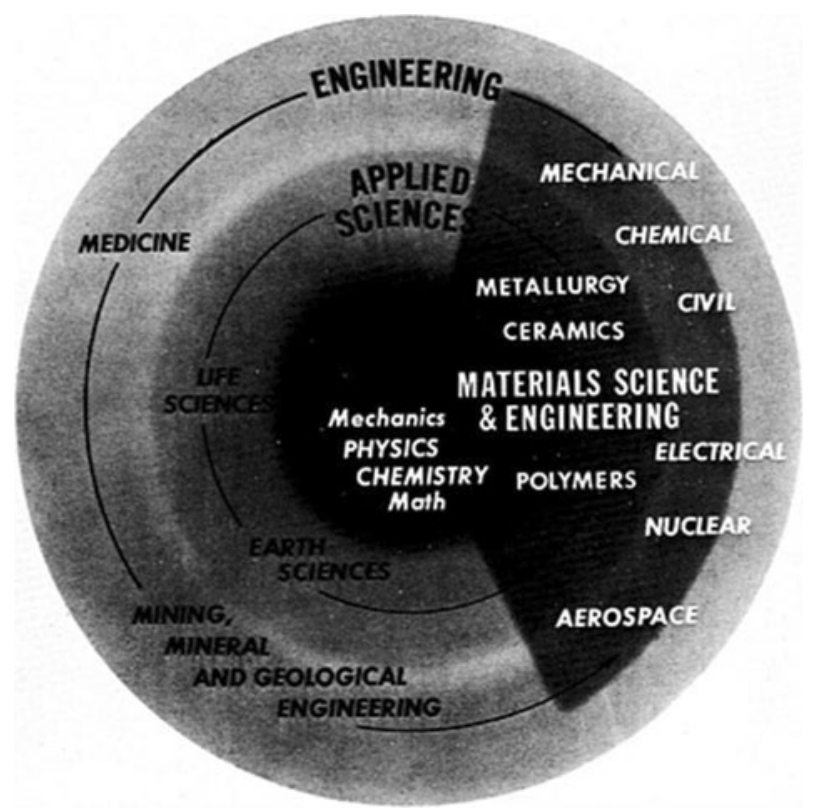

Abb. 5 Multi-discipline materials science and engineering (COSMAT 1974: 26) 


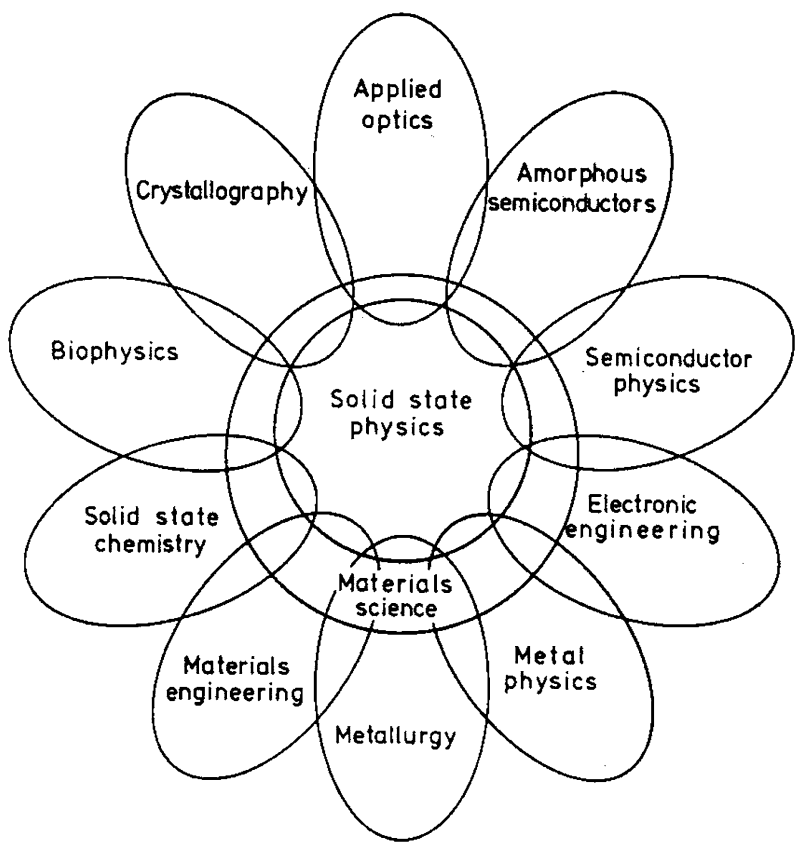

Abb. 6 Eine physikalistische Vision von materials science/s als Festkörperwissenschaft (solid state science) (Weaire/Windsor 1987: x)

Kristallographie, angewandter Optik, Elektronik, Metallphysik, statistischer Mechanik und so weiter, aus denen sich das andere sicherlich ergäbe: „Solid State Physics is at the core of nearly all [these] fields, while Materials Science embraces the wider application of the basic studies." (Weaire/Windsor 1987: x) Leider zeichnete diese Vision zweier Physiker ein falsches Bild: Festkörperphysik kam historisch (zu) spät, war zu lange zu stark auf die retrodiktive Erklärung bereits beobachteter Eigenschaften von Materialien fokussiert und hatte sich zu wenig mit der Voraussage und Konstruktion neuer Materialien beschäftigt. ${ }^{34}$

Eine weitaus differenziertere Konzeption entwickelte Rustum Roy (19242010), Gründer des Materials Science Laboratory an der Pennsylvania State University, in dem von ihm herausgegebenen Band zum Stand von MSE (Roy 1970). Er situiert darin materials research als eine Art Kontaktzone, eine Art Vorgriff auf Galison's trading zones, zwischen den älteren disziplinären Nachbarn aus Natur- und Technikwissenschaften, wobei er allen diesen Disziplinen durchaus zugesteht, ebenso wie die „neodiscipline“ MSE Materialforschung zu betreiben. An MSE, in Abbildung 7 an Stelle von Festkörperphysik ins Zentrum gerückt, sei das Besondere, dass nur materials research betrieben werde; deswegen die gestrichelten Linien, die aus dem dick umrandeten Zentrum der materials research wegführen. 


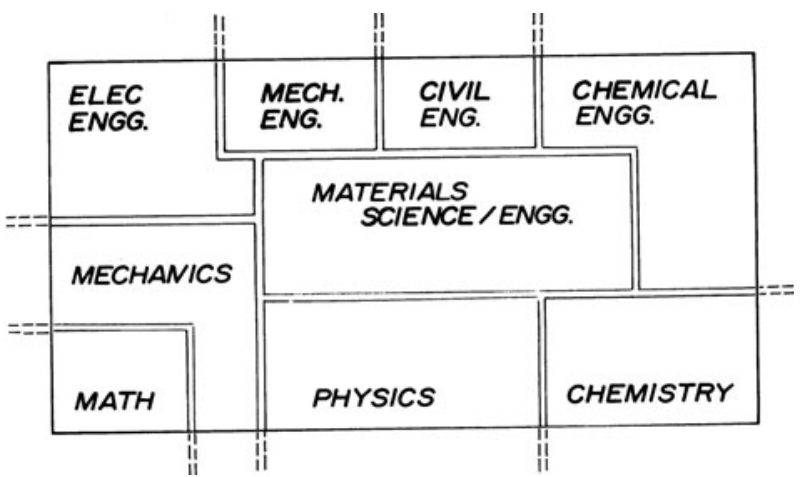

Abb. 7 MSE im Zentrum von materials research hier als fest umrandete Kontaktzone verschiedener älterer Disziplinen (Roy 1970: 161)

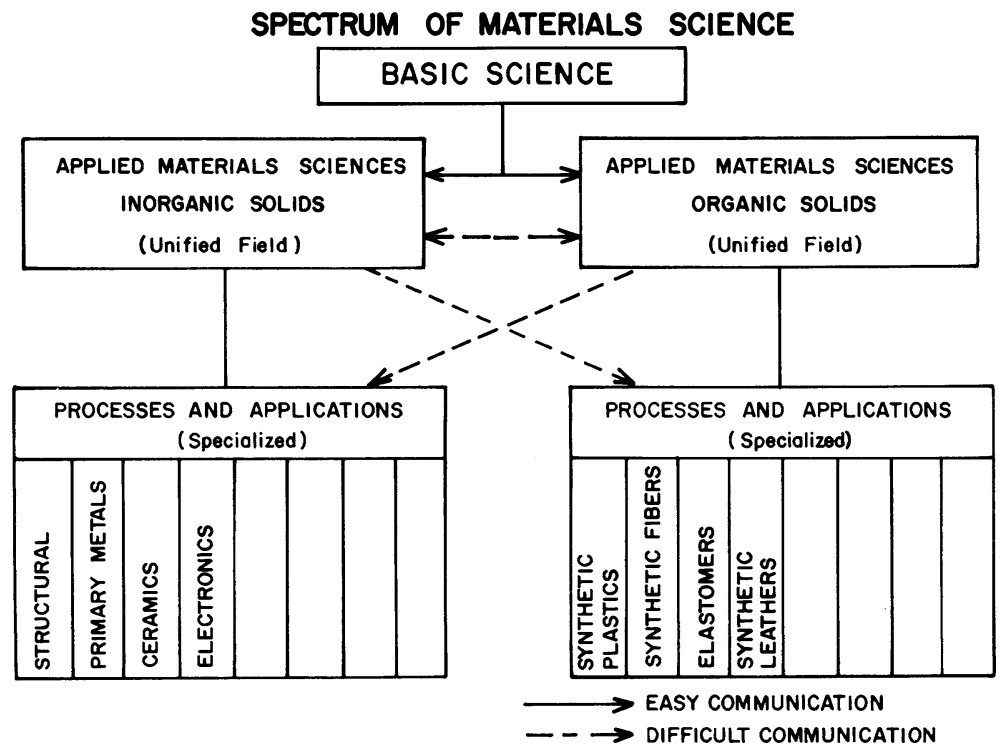

Abb. 8 Darstellung der Disziplinbeziehungen durch Krauss (1970: 41)

Die damit schon angesprochene Frage nach kommunikativen Querverbindungen zu den disziplinären Nachbarn ist ein anderes Diagramm des gleichen Sammelbands gewidmet, das vom Metallurgen George Krauss ("1933) von der Lehigh University entworfen wurde (vgl. Abb. 8). Als wichtigste kommunikativ noch nicht geschlossene ,innere Front' der MSE wird hier die Spaltung in organische und anorganische Werkstoffe gesehen; das ,lineare Modell' wird auch hier offenbar unproblematisch vorausgesetzt und sogar als Hierarchie von oben-unten wiedergegeben, wobei die Top-down Kommunikation zwischen 
Grundlagen- und Anwendungsforschung als unproblematisch eingeschätzt wird.

\section{Indikatoren für und gegen eine Konvergenz zur Disziplin}

Werden die von Akteuren gemachten Vorschläge zu MSE als Funktion der Zeit darstellt, so ergibt sich eine beträchtliche Bandbreite von Möglichkeiten mit einer interessanten Tendenz:

- „a new synthesis of disciplines“ (Harwood 1970:1)

- „coherent aggregation“ of related activities (Calvin 1973)

- „multi-discipline“ (COSMAT 1975; 1989; Flemings 1999: 16-19)

- "the array of disciplines and subdisciplines that overlay the materials cycle" (Cohen 1979: viii)

- „many disciplines involved [...] but multidisciplinary cohesion“ (Kranzberg/ Smith 1979: 33)

- „superdiscipline“ (Turnbull 1983: 2)

- „Materials is not to be thought of as a single discipline, but rather as a broad and vital field of knowledge and techniques" (Johnson 1987)

- „ultramolecular“ (Turnbull 1983: 1) beziehungsweise „Supramolecular Science" (neue Zeitschrift 1995)

- „a very loose alliance of recognized subdisciplines such as Metallurgy, Continuum Mechanics, Crystallography, Polymer Chemistry, and Solid State Physics. Or one might think of it as a confederation of innumerable research specialities“ (Ziman 1995: 2047)

- „Is materials science a proper discipline or is it like a tangle of jungle creepers, hanging heavily between disciplinary trees? [...] just a multidisciplinary chimera?" (Cahn 1997: 160)

- „it is time to view the core of our field as a discipline itself“ (Flemings/Cahn 2000: 380)

- „a discipline (while simultaneously part of a larger discipline)“ (ebd.: 371)

- „an independent interdisciplinary science“ (vgl. European White Book, Rühle 2002:115)

- „cross-cutting area between scientific research and industrial development" (Stratmann 2006)

Es fällt auf, dass das erste klare Votum für materials science als neue, eigene Disziplin erst spät, um das Jahr 2000 auftaucht und dass dessen Hauptvertreter, die Materialforscher Merton C. Flemings ("1929), der von 1980 bis 1982 erster Direktor des Materials Processing Center am MIT war, und Robert W. Cahn (1924-2007), der an der University of Sussex von 1965 bis 1981 die erste britische Professur für materials science innehatte, sich ihrer 
Sache noch so wenig sicher waren, dass sie an anderer Stelle der gleichen Publikation auch eine Gegenkonstruktion mitanbieten. Aufgrund dieser Vermischung von Rhetorik und Vision, überzogenen Anspruchshaltungen und nicht eingelösten Erwartungen empfiehlt es sich, für die nun anstehende Prüfung dieser Ansprüche auf die in der Wissenschaftsforschung anerkannten, systematischen Kriterien zurückzugreifen, um festzustellen, ob eine Disziplin vorliegt oder nicht. Gehen wir die in der Einleitung vorgestellten Indikatoren am Beispiel der materials science sowie der Material- beziehungsweise Werkstoffwissenschaft durch, so sehen wir rasch, dass diese mit Ausnahme des ersten Punktes (Paradigmen und durchgängige Methoden), zum jetzigen Zeitpunkt allesamt vorliegen.

Um beim Indikator, Vorliegen einschlägiger wissenschaftlicher Zeitschriften' zu beginnen: Im deutschen Sprachraum gibt es eine dominante Zeitschrift, und zwar die Internationale Zeitschrift für Metallographie, 1909 als Zentralblatt für die gesamte Theorie und Praxis der Metalle und Legierungen von William Guertler (1880-1959) gegründet, der damals Privatdozent an der TH Berlin-Charlottenburg, ab 1931 Mitglied der NSDAP und ab 1933 ordentlicher Professor für Metallkunde war. Aus diesem Zentralblatt entstand 1919 die Zeitschrift für Metallkunde, so umbenannt aufgrund der ebenfalls im selben Jahr erfolgten Gründung der Deutschen Gesellschaft für Metallkunde. ${ }^{35}$ Bis in die 1930er Jahre waren Wissenschaft und Technik gleich stark vertreten, danach nahmen naturwissenschaftliche Beiträge stark zu, bei gleichzeitigem Rückgang der technischen Beiträge. 1992 erhielt die Zeitschrift einen zusätzlichen englischen Untertitel, der mit der Internationalisierung der Trägergesellschaft zusammenhängt: International Journal of Materials Research. Mit dem 97. Band im Jahre 2006 wurde er zum Haupttitel und inzwischen erscheinen alle Beiträge in englischer Sprache. Daneben gibt es eine Vielzahl weiterer, großteils englischsprachiger Zeitschriften für Materialwissenschaftler, so etwa Materials Science and Engineering (erschienen seit 1966, heute in mehreren Unterreihen), ferner das Journal of Materials Education, das International Journal of Educational Modules for Materials Science and Engineering (seit 1979 herausgegeben von Mitarbeitern des Materials Research Laboratory an der Pennsylvania State University), und die Acta Metallurgica (seit 1954 bestehend). Letztere wurde seit circa 1990 in Acta Metallurgica et Materialia und seit 1974 in Materialwissenschaft und Werkstofftechnik umbenannt, um nur vier wichtige weitere Beispiele zu nennen. ${ }^{36}$

Sowohl auf der Ebene der Fachgesellschaften wie auch auf der von Instituten und Lehrstühlen finden sich in den beiden Jahrzehnten zwischen 1970 und 1990 zahlreiche Beispiele für Umbenennungen, in denen ,Metall' durch ,Material' ersetzt wurde. Ein typisches und zugleich historisch wichtiges Beispiel dafür ist das ehemalige Göttinger Institut für allgemeine Metallkunde, das auf den noch als physikalischen Chemiker ausgebildeten Metallurgen Gustav Tammann (1861-1938) zurückgeht, ${ }^{37}$ und jeweils mit dem Wechsel des 
Göttinger Lehrstuhlinhabers zwischen 1970 und 1993 folgende Serie von Umtaufungen durchlaufen ist: erst Institut für Metallphysik und allgemeine Metallkunde; dann Institut für Metallphysik und schließlich seit Ende der 1990er Jahre: Institut für Materialphysik. Analog dazu, wenngleich zeitlich etwas früher, fanden die Umbenennungen des Department of Mining and Metallurgy am MIT statt. Dieser Fachbereich hatte unter dieser Bezeichnung dort seit 1888 bestanden. 1967 wurde er in Department of Metallurgy and Materials Science und 1974 in Department of Materials Science and Engineering umbenannt. ${ }^{38}$ Am Department of Materials Science and Metallurgyin dieser Reihenfolge! - der University of Cambridge wurden seit 1976 die Prüfungen der von 1966 bis 1976 noch getrennten Institute of Metals beziehungsweise Institute of Materials zusammengefasst (Charles/Greer 2005). Die Deutsche Gesellschaft für Metallkunde wurde 1989 auf Initiative von Werner Köster $^{39}$ (1896-1989) in Deutsche Gesellschaft für Materialkunde umbenannt. Analog hieß die französische Gesellschaft Société Française de Métallurgie et de Matériaux. Das 1908 gegründete Londoner Institute of Metals wurde 1976 zunächst in Institute of Materials und 2002 dann in Institute of Materials, Minerals and Mining umbenannt. In den USA gründete sich 1973 die Materials Research Society und in Europa mit der auch sonst typischen Verspätung von etwa einem Jahrzehnt 1983 die European Materials Research Society und bald darauf die International Union of Materials Research Societies. 2002 erschien das erste White Book of the European Union on Fundamental Research in Materials Science und 2009 ein weiteres Weißbuch speziell zur nanomaterials science (Dosch/van der Voorde 2009, Schummer 2011).

Ein weiterer Indikator für die zunehmende Institutionalisierung des Feldes ist die in dieser Phase der 1980er und 1990er Jahre zunehmende Tendenz, vom Plural ,materials sciences' zum Singular ,materials science' überzugehen, was nicht mehr den losen bunten Strauß von Teilaktivitäten evoziert, der in Abbildung 6 zu sehen war, sondern eine geeinigte, kohärent gewordene Disziplin, etwa im Sinne der Darstellung in Abbildung 7. Unter diesem Vorzeichen standen auch der Ausbau einschlägiger Forschungsaktivitäten in Universitäten und Industrie sowie von Neubauten für thematisch pertinenter Forschungscluster, die Einrichtung von Diplom-, Bachelor- und Masterstudiengänge mit diesen Bezeichnungen und vermehrt auch von entsprechenden Lehrstühlen. Seit 2000 erfolgte auch in Deutschland die Umbenennung von chemischen beziehungsweise physikalischen oder ingenieurwissenschaftlichen Instituten und Studiengängen.

Dieses erhebliche quantitative Wachstum des Feldes kann hier mit einigen Zahlen untermauert werden: bis 2000 wurden allein in den USA 41 reine MSEDepartments eingerichtet, zu denen 14 spezialisierte und weitere 41 zumindest teilweise diese Themen abdeckende Departments in Physik-, Chemieoder ingenieurwissenschaftlichen Umfeldern hinzukommen. In den USA wurden zwischen 1990 und 1994 jährlich etwa 1100 BA-Abschlüsse in MSE 
gemacht; dementsprechend verfügte um 2000 etwa ein Drittel aller Mitarbeiter an entsprechenden Projekten über fachspezifische Abschlüsse. Es gab 1997 circa 625 festangestellte Lehrende in MSE. Auch in anderen Ländern ist die Situation grundlegend ähnlich: Bis 1998 waren in England 21 reine Departments für MSE eingerichtet worden, unter Zuzählung der nur Teilgebiete abdeckenden Departments kommt man auf insgesamt circa 61, in Deutschland auf circa 79, in Italien auf 72, in Frankreich auf 48, in Polen auf 50, in der Schweiz auf sieben und in Schweden auf vier. ${ }^{40}$ Große Anstrengungen in Richtung der Materialwissenschaft wurden auch in Japan unternommen, wo neben einem 2001 gegründeten National Institute of Materials Science (das aus dem seit 1956 bestehenden National Research Institute for Metals hervorgegangen ist) das National Institute of Advanced Industrial Science and Technology und das ältere und stärker grundlagenforschungsorientierte Institute of Physical and Chemical Research sowie zahlreiche kleinere Forschungseinheiten Materialforschung betrieben wird.

Eine Übersicht über die Vielzahl heute alleine in Deutschland auf Gebieten der MSE tätigen Forschungsinstitutionen und deren relative Nähe oder Ferne zu den drei großen Polen der MSE, das heißt den Werkstoffwissenschaften, der Physik und Chemie sowie den Ingenieurwissenschaften, Maschinenbau und Elektrotechnik, wird in Abbildung 9 gegeben: Auffallend ist in dieser Darstellung von Mitarbeitern der Deutschen Gesellschaft für Materiaforschung, dass nur die wenigsten dieser Institute, etwa in Chemnitz oder in Dresden, von den materialwissenschaftlich arbeitenden Akteuren selbst in einer ausgewogenen Mitte zwischen diesen drei Polen situiert wurden, während das Forschungsprofil der großen Mehrzahl von Instituten nach wie vor sehr nahe an einem der Gebiete angesiedelt sind. Die Vorstellung einer durchgängigen materials science war noch nicht vorhanden. Zumindest in Deutschland wurde 1994 noch kein Disziplinenanspruch erhoben.

Wenn Robert W. Cahn also mit seiner seit 2000 erhobenen Behauptung Recht hätte, dass es Zeit sei, materials science als neue Disziplin zu betrachten, so würde es sich hier um das seiner eigenen Aussage nach seltene, aber keineswegs singuläre Muster einer „emergence by integration“ handeln. Ob materials science tatsächlich eine vollwertige Disziplin darstellt, ist aber noch heftig umstritten. In seinem Lehrbuch fragt Cahn (2001a: viii, 1997: 161) bezeichnenderweise auch selber: "Can a discipline also be interdisciplinary? Is materials science a real discipline?" Als wichtigstes Indiz für eine Konvergenz führen Cahn und Flemings die zunehmende Integration der beiden grössten Bereiche an, die lange Zeit eigenständig Forschung betrieben hatten:

Metallurgy and ceramics are fast becoming absorbed in our thinking into the broader field of MSE, just as nonferrous metallurgy, ferrous metallurgy and extractive metallurgy became subsumed in metallurgy a generation or more ago [...] the rationale [for viewing the core of our field as a discipline itself, K.H.] is the intellectual cohesiveness the field has developed over the past 30 years, the 


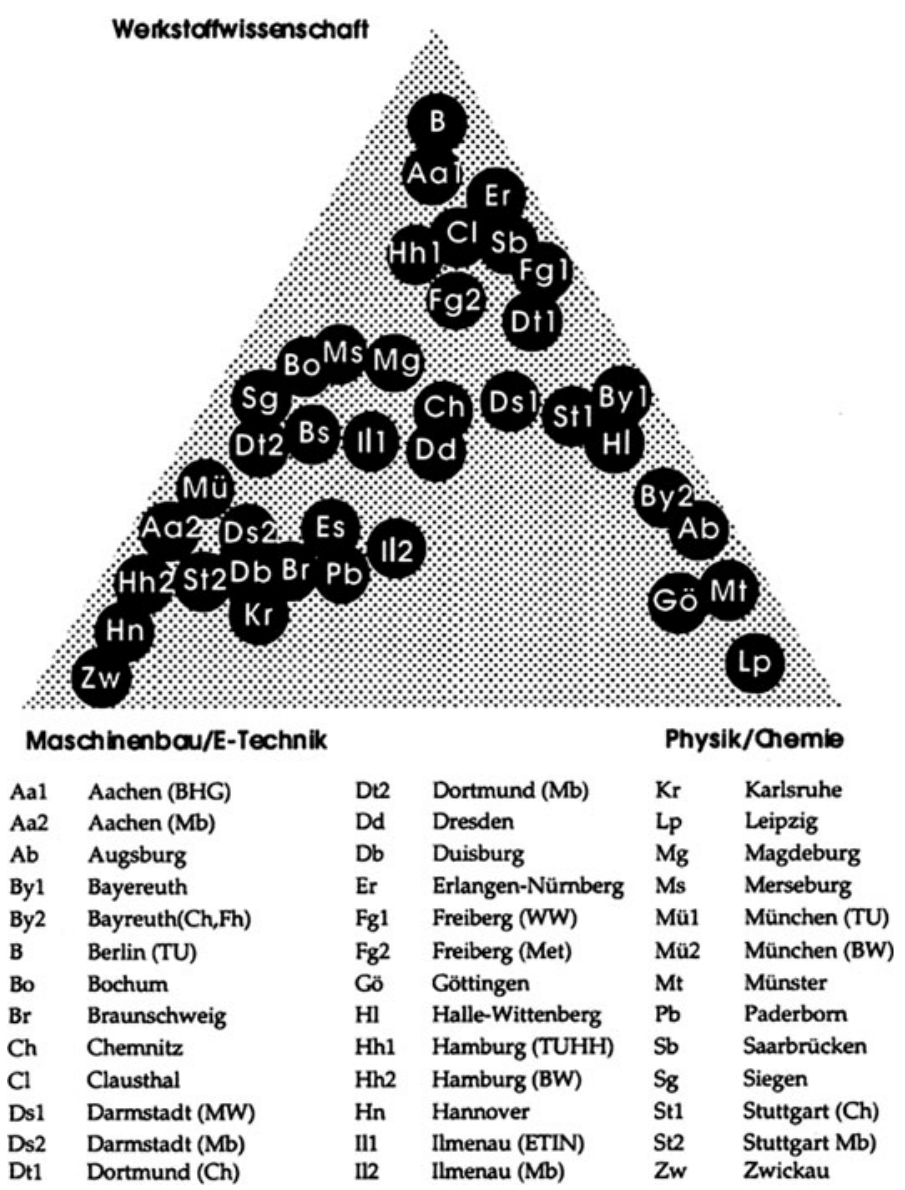

Abb. 9 Schwerpunktverteilung an 40 deutschen MSE-nahen Instituten (Deutsche Gesellschaft für Materialforschung 1994: 8)

industrial recognition it increasingly enjoys over what are becoming the narrower sub-disciplines of metallurgy, ceramics, polymers, etc. (Flemings/Cahn 2000: $380)^{41}$

Daneben nannten die beiden Autoren einen weiteren, aus der Literatur zur Disziplinformation ebenfalls wohlbekannten Indikator, nämlich die beginnende Innen-Aussen-Demarkation, von Soziologen auch als Abgrenzungsarbeit (boundary work) bezeichnet (Gieryn 1983, 1995; Reinhardt 2011):

We distinguish ourselves from our peers in chemical engineering (who have largely taken over extractive metallurgy) by our expertise in structure and structure-property relations as well as our breadth across materials classes. [...] We similarly distinguish ourselves from mechanical engineering by our focus on the internal structure of materials, specifically on atoms and molecules. [...] Our fundamentals are... structure, processing, properties and performance. We emphasize relationships among these elements more than other academic departments. (Flemings/Cahn 2000: 381) 
Die mehrfach wiederholte Verwendung der dritten Person Plural signalisiert ein vorhandenes Gruppenbewusstsein. Das „we distinguish ourselves from our peers" war darüber hinaus eine klare explizite Abgrenzung gegenüber den disziplinären Nachbarn, die als Konkurrenten um Studenten, Ressourcen und Ideen begriffen wurde, gegen die MSE lanciert wurde. ${ }^{42}$

Die "fundamentals“ im letzten Satzes des Zitats spielen auf die sogenannten vier Elemente der Materialwissenschaft an (COSMAT 1989), die in Lehrbüchern seither gerne in Form eines Tetraeders abgebildet werden (vgl. Kranzberg/Smith 1979: 31, Flemings 1999: 2). Sie zeigen die vier in sehr verschiedene Richtung gehenden Anliegen der Materialwissenschaft von Strukturaufklärung bis Produktion in trauter Eintracht und Harmonie vereint in einem der Platonischen regelmäßigen Körper (vgl. Abb. 10). Je nach Herkunft und Prioritäten des jeweiligen Autors findet sich manchmal auch structure/composition oben auf der Spitze, aber allen ist klar, dass MSE diese vier Komponenten in harmonischem Verhältnis und in fruchtbarer Wechselwirkung miteinander beinhalten muss, um erfolgreich zu sein. Die Einigung auf ein solches Diagramm war ein eher symbolischer, aber wichtiger Schritt zur Findung des gemeinsamen Paradigmas.

Früher, so Robert W. Cahn (2002) in seinem launigen Auftaktsbeitrag zum ersten erschienenen Heft von Nature Materials, mit dem gleich ein weiterer Indikator der vollendeten Institutionalisierung vorliegt, hätten sowohl von Seiten der Grundlagenwissenschaften Physik und Chemie als auch von Seiten der Ingenieurwissenschaften große Vorbehalte gegen eine eigenständige Disziplin der Materialwissenschaft bestanden. Man denke nur an das Naserümpfen gegen Sommerfelds „Schmierölphysik“ (Eckert 1993) oder Wolfgang Paulis entsetzte Reaktion auf Rudolf Peierls vorsichtigen Vorschlag, doch einmal eine Theorie elektrischer Leitung in Festkörpern zu unternehmen: „One shouldn't wallow in dirt" (Cahn 2002: 4). Doch mit der rasanten

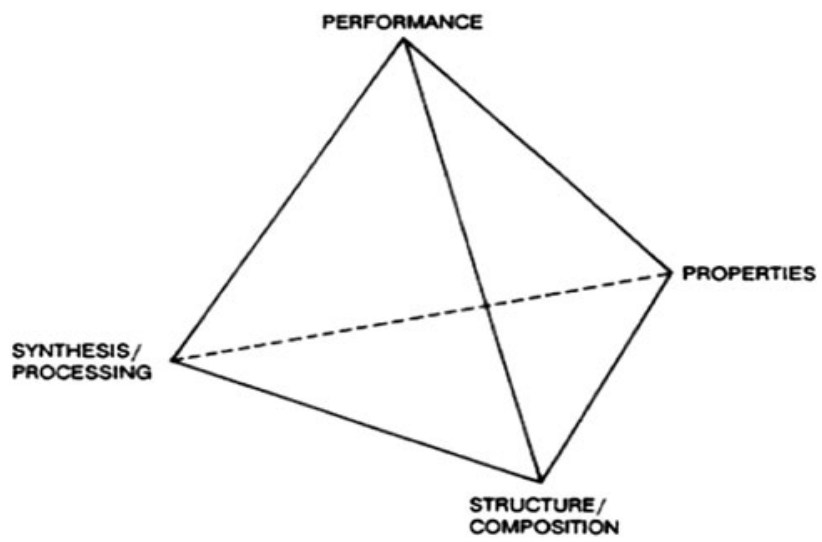

Abb. 10 Die vier Elemente der MSE (COSMAT, 1989: 29) 
Entwicklung der Halbleiterphysik und der durch sie möglich gemachten Revolution der Mikroelektronik (Queisser 1985, Riordan/Hoddeson 1997) verwandelte sich dieses Vorurteil gegen Verunreinigungen und Abweichungen von idealen Kristallgittern in ein zunehmendes Interesse an eben diesem Einfluss kleinster Beimengungen, die nunmehr mit positiver Konnotation ,Dotierung' genannt wurden. Heute ist die gezielte Kontrolle und Beeinflussung solcher Dotierungen in parts per trillion und andere mikrostrukturelle Besonderheiten eine der Kernaufgaben der Materialwissenschaft als eine "science of dirt" geworden (vgl. Cahn 2002, Schummer 2011). Jedoch blieben wenigstens fünf Indizien, die - zumindest in den Augen von Bensaude-Vincent 2001 - gegen eine Konvergenz zur Disziplin sprechen:

Erstens verwirrt nach wie vor die erhebliche Themenbreite und Methodenvielfalt (je nach speziellem Anwendungsgebiet, reichend von photonischen und magnetischen Materialien über Keramiken bis zu Composite-Materialien (Canby 1989, Sass 1998: Kap. 14, Haka 2011, im Druck). Zweitens besteht ein geringer Kernbestand an Wissen, der in allen Textbüchern des Feldes, mit spezifischen Eintönungen je nach Forschungsrichtung vor Ort vermittelt wird. Drittens gibt es wenige kanonische Lehrgegenstände, darunter insbesondere viele Importe aus der Kristallographie- und Festkörperphysik wie Gitter, Fehlstellen und Oberflächenstrukturen. Viertens sind unter anderem Polymere komplexer Chemie in der MSE nach wie vor eher marginal, wobei insbesondere die Polymerchemie eigenständig in der Chemie institutionalisiert ist ${ }^{43}$ und fünftens überwiegt nach wie vor das Selbstverständnis der Akteure als Angehörige eher traditioneller Fächer, also zum Beispiel als Festkörperphysiker, Polymerchemiker, Ingenieur. ${ }^{44}$

Unter diesem Eindruck geringer inhaltlicher Kohärenz kam BensaudeVincent, die sich am längsten mit der Geschichte der Materialwissenschaften beschäftigt hat, zu einem ablehnenden Gesamturteil in der Frage disziplinärer Einheit der Materialwissenschaft:

The overall impression is one of an aggregation of fragments of knowledge taken from various disciplines around a core of atomic physics [...] no inner logic presides over the organization of the discipline forty years after its emergence. Rather, the organization of most textbooks simply reflects the successive layers of the field's historical development [... there is a] clustering of specialists from various disciplines and an amalgamation of science and technology. ${ }^{45}$

Nahezu ein Jahrzehnt später komme ich bei erneuter Inbetrachtnahme aller Indikatoren für Disziplinenentwicklung zu einem anderen Urteil: Es bestehen einschlägige und einflussreiche internationale und nationale Fachgesellschaften, Lehrstühle und ganze Institute mit dieser Denomination, ebenso etliche Periodika, die die ganze Breite der materials science und nicht nur spezielle Teilmengen davon umfassen, ferner zunehmend im Fach anerkannte, integrative Lehrbücher und Studiengänge. Längst gibt es auch ein invisible college von Personen im Kern des neuen Fachs, die sich regelmäßig auf 
Tagungen und Strategietreffen sehen, um Forschungsprogramme, neue Schwerpunkte zu koordinieren und rhetorisch ebenso wie sozial und institutionell eine aktive Abgrenzungsarbeit zu konkurrierenden Nachbarfeldern und zu den Mutterdisziplinen zu betreiben. ${ }^{46}$

Auch in Bezug auf den lange Zeit problematischsten Indikator, die Formierung von verbindlichen Paradigmen, hat das Forschungsfeld im letzten Jahrzehnt große Fortschritte gemacht. Neue Lehrbücher wie etwa das der beiden MIT-Autoren Samuel M. Allen (Professor of Physical Metallurgy am dortigen Stevens Institute of Technology) und Edwin L. Thomas (Head of the Department of Materials Science and Engineering) sind konsequent auf einem durchgehenden Satz ,struktureller Deskriptoren' aufgebaut, die allgemein für die Charakterisierung der verschiedensten Stoffklassen geeignet sind, so zum Beispiel morphologische Fehlstellen wie Leerstellen, Versetzungen, Korngrenzen oder Poren, veränderte Ordnungsparameter bei Phasenübergängen, ferner zum Beispiel Diffusion oder andere zufallsgesteuerte Prozesse, die von den Autoren in Kristallen, Gläsern, Keramiken und Flüssigkristallen gleichermaßen exemplifiziert werden. ${ }^{47}$ Auch in dem eingangs erwähnten, historisch aufgezogenen Lehrbuch von Rolf E. Hummel ("1934), der am College of Engineering der University of Florida gelehrt hat, demonstrieren die ausgewogene Betrachtung der verschiedensten Stoffklassen und die Integration dieses Wissens. Korrelativ zu dieser Verwissenschaftlichung und Institutionalisierung ist die zunehmende Ausgrenzung der nur anwendungsund praxisbezogenen Techniken als bloßer ,materials technology ${ }^{48}$ und die Aufgabe der früher allgegenwärtigen Abkürzung MSE. Von anderen Autoren wie etwa dem Stuttgarter Spezialisten für Hochleistungskeramiken, Fritz Aldinger, der am Stuttgarter MPI für Metallphysik die Abteilung für Materialsynthese und Gefügedesign und zugleich an der Universität das Institut für nichtmetallische anorganische Materialien leitet, wird die spezifische Situierung der Materialwissenschaft zwischen den Natur- und den Technikwissenschaften eher in der von ihren Modellierungen, Experimenten und zunehmend auch von Simulationen erfassten Mesoskala der Materie in typischen Größenordnungen von $10^{-8} \mathrm{~m}$ bis $10^{-3} \mathrm{~m}$ gesehen (Aldinger/Baumard 2002: 28, 1999: 34). Bei noch kleineren Skalen greift die in den 1990er Jahren einsetzende Rhetorik der nanoscience and nanotechnology (vgl. Schummer 2011), die derzeit ebenfalls um Institutionalisierung ringt.

Freilich bleibt das neue Feld der materials science, das Metalle, Halbmetalle, Legierungen und Nichtmetalle, Gläser und Keramiken, Polymere und Composite-Materialien sowie unlängst zunehmend auch Biomaterialien zu umspannen versucht, unübersichtlich und vielgestaltig, was selbst die Redakteure von Nature Materials im ersten Heft 2002 zugaben. Dort wird von einem ",culture gap between scientists involved in research into ,soft' and ,hard' matter" gesprochen, wobei eingestanden wird: „The diversity and ,frontier' spirit can sometimes be problematic." (Cahn 2002: 1) Noch verbleibende 
Akzeptanzprobleme werden auf die Ursprünge ihrer Disziplin in den Technikwissenschaften zurückgeführt. Im Editorial des zweiten Jahrgangs von Nature Materials ist unter der dramatischen Überschrift: „A matter of survival" folgender Appell an die Materialwissenschaftler zu lesen, sich dem Niedergang der traditionellen materialintensiven Industrien im Bereich Metallurgie und Aerospace durch stärkere thematische Diversifizierung in allen Materialklassen, gekoppelt an disziplinäre Einigung, zu entziehen:

Most materials science departments in the US and UK have their roots in nineteenth century metallurgy and/or mining departments. If they are to flourish and justify their existence in years to come they will need to shake off their traditional image as engineering departments and adapt to new industrial and societal needs. [...] the need to educate students not only across all materials classes [...] but also across other traditional disciplines [...] is increasingly putting pressure on the materials science curriculum. One solution to this problem may be that, although the field should remain a multidiscipline, it should be thought and taught as a coherent and single discipline. (Dusastre 2003: 1)

In seiner Antwort betonte Cahn (2003a), dass dieses schon geschehe. MSE hat sich vielerorts des Zusatzes ,engineering' entledigt und ist zur ,materials science' geworden. Sie hat sich somit im Licht der eingangs aufgelisteten anerkannten Indikatoren für das Vorliegen wissenschaftlicher Disziplinen als ebensolche erwiesen. Das Muster, nach dem dieses geschah, unterschied sich jedoch grundlegend von der typischen Disziplinenausdifferenzierung, wie wir es aus dem 19. und frühen 20. Jahrhundert kennen (Stichweh 1984). Das Institutionalisierungsmuster der Materialwissenschaft entspricht definitiv nicht der klassischen Disziplinengenese, etwa der Physik und der Chemie (um 1800) oder dem der physikalischen Chemie (um 1900). ${ }^{49}$ Doch spricht dies nicht gegen den Disziplinenstatus der materials science.,Transdisziplinarität' wird innerhalb der MSE beziehungsweise der Werkstoffwissenschaft eben nicht in Anspruch genommen, sondern bestenfalls eine ,Multidisziplinarität' (wie etwa im letzten Zitat), oft aber auch mit dem seit 2000 von Akteuren immer wieder erhobenen Anspruch auf eine eigene disziplinäre Identität gekoppelt. Diese Selbstverortung erfolgt durchaus aus systematisch nachvollziehbaren Gründen: So liegen stabile Institutionen, Zeitschriften, kanonische Lehrinhalte und erfolgreiche Lehrprogramme vor, die die systemische Reproduktion der Materialwissenschaft ermöglichen und seit den 1990er Jahren ist zunehmend auch die bewusste Abgrenzung und Konkurrenz zu anderen Disziplinen um pekuniäre und instrumentelle Ressourcen sowie um den wissenschaftlichen Nachwuchs zu beobachten. ${ }^{50}$ Die bis heute so unbequeme Grenzstellung der Materialwissenschaft zwischen tradierten disziplinären Großfeldern, insbesondere auch zwischen Natur- und Technikwissenschaften, erklärt vielleicht auch, warum die Geschichte dieses Forschungsterrains bislang von einer ebenfalls entlang dieser Bereiche 
aufgeteilten Historiographie der Physik, der Chemie und der Technik so wenig in den Blick genommen wurde.

\section{Anmerkungen}

1 Smith 1961, 1969, 1977, 1986a oder b zw. Cahn 2001a. Für von Materialforschern selbst zusammengestellte Dokumentationen wichtiger Etappen: Smith, Hg, 1965, 1968, Tylecote [1976] 1992, Psaras/Langford, 1987. Weaire/Windsor 1987, Sass 1998, Wagner 2000; allgemeine Überblicke ferner in Cunliffe, Hg., 1994, Dresselhaus 1992, Strahm 1995, Maier 2000, Wagner 2000, Braun/Herla, Hg., 2002.

2 Siehe Chubin 1976, Lemaine, 1976, Stichweh 1984, Thiel/Peckhaus 1999, Laitko 2002.

3 Einen nach Stoffklassen getrennt jeweils historischen und systematischen Zugang kombiniert Rolf Hummel in seinem 1998 erschienenen Lehrbuch. Zum Naturwerkstoff Holz: Radkau 2007; zu Biomaterialien: Geison/Creager 1999; zu Keramiken: Mämpel 1985, Kingery 1986, Aldinger 1999; zu Polymeren siehe Anmerkung 43.

4 Cahn 2001b: 307, von ihm ausdrücklich als Gegenmodell zur traditionellen Disziplinenbildung des 19. Jahrhunderts eingeführt, die durch Ausdifferenzierung erfolgte (vgl. hier Anm. 39). Andere Kandidaten für „discipline formation by integration“ sind Geologie und Molekularbiologie sowie computer science (Ceruzzi 1989). Smith 1986b: 3 schlägt eine interessante Analogie zur „aggregation of electrons into atoms and matter" vor.

5 Réaumur 1722; Leon 1912: 13-16, Smith 1969: 483-487, Tylecote [1976] 1992: 177. Zu den Umbrüchen der Materialkategorien in der Chemie unter anderen Experimentalwissenschaften im 18. Jahrhundert: Klein/Lefèvre 2007 Bensaude-Vincent 2011.

6 Day 1995 geht so weit, Faraday retrospektiv als „materials scientist“ avant la lettre zu interpretieren.

7 Man denke etwa an die Spiegel-Meßgeräte von August Wöhler, Adolf Martens, Ernst Preuß unter anderem zur Bestimmung der Feindehnung von Materialien unter Belastung oder an die auf Ludwig Werder (1808-85) zurückgehende Universalprüfmaschine, beschrieben zum Beispiel in Baumann 1912, Domke [1964] 1999: 290 f., Nüßlein 1965, Opitz/Dude [1964] 1972: 53 f., Kloos/Berger 2007: 312-314.

8 Ramsauer 1943, Qing 1995, Rasmussen 1997, Newbury/Williams 2000.

9 Binnig/Rohrer 1985, 1987, Wickramasinghe 2000, Hennig 2004, 2009, Mody 2006, Granek/Hon 2008; weitere Beispiele in Ashby u.a. 1980, Cockayne 2002, Mody 2006.

10 Über diese Transgression der ursprünglichen Disziplinengrenze und die damit verbundenen Transformationen des Forschungsstils: Reinhardt 2006, 2011.

11 Sorby 1897, Mehl 1948: 11-13, Higham 1963, Smith, Hg, 1965, 1969: 491 f., Hammond 1989, Tylecote 1992: 180, Edyvean/Hammond 1997.

12 Siehe z.B. Martens 1880, 1898 sowie Piersig 2007, 2008 und Werner 1964; ein weiterer Meilenstein in der Durchsetzung der Metallographie war das Lehrbuch von Ernst Preuß, das 1913 erschien (Preuß [1913] 1927). Preuß war Schüler von Martens und ab 1907 Assistent an der Darmstädter mechanisch-technischen Materialprüfungsanstalt.

13 Bach/Baumann [1914] 1921, mit nahezu 1000 schwarz-weiß Abbildungen zu den verschiedensten Materialfehlern illustriert, sowie den Atlas Metallographicus von Hanemann/Schrader [1933] 1952; vgl. Baumann 1920, Pusch 1979.

14 Siehe zu historische Dampfkesselexplosionen und Eisenbahnunfällen, die ihre Ursache in Materialfehlern oder Materialermüdung hatten: Sonnenberg 1968, Krankenhagen/Laube 1983.

15 Wöhler 1877, Bauschinger 1879, vgl. Schulz 1949, Kloos/Berger 2007: 311. Die Bedeutung der Vereine für die Konsolidierung der Materialforschung betont Helmut Maier.

16 Siehe Baumann (1912: 154; 177-184) zu der seit 1895 steil steigenden Zahl von Prüfungsmaschinen und -vorgängen.

$17 \mathrm{Zu}$ den Materialprüfungsanstalten siehe zum Beispiel Föppl 1959, Amstutz 1955, Zweckbronner 1985, Blind/Werner 1984, Ditchen 2009, Kloos/Berger 2007; weitere 
nichtpreußische Materialprüfungsanstalten werden vorgestellt in: Anonym 1930, Sonnemann/Friedrich 1978; zum Berliner königlichen Materialprüfungsamt siehe Martens/ Guth 1904, Ruske 1971, Ruske/Becker/Czichos 1996; zum American Institute of Mining and Metallurgical Engineers (gegr. 1871): Mehl 1948; zum Aufschwung der Werkstoffprüfung im 19. Jahrhundert allgemein: Schulz 1949, Sigwart 1986, Kahlow 1991.

18 Ein geeignetes Beispiel dafür sei das für Ingenieurschulen der DDR verbindliche Lehrbuch von Opitz/Dude genannt, das zwischen 1964 und 1977 in sieben Auflagen erschien; ein westdeutsches Beispiel sind die diversen Auflagen von Domke 1964.

19 Zu deren neuen Materialklassifikationen und Prüfinstrumenten, siehe Föppl 1959, Kahlow 1991: 299 f., Krankenhagen/Laube 1983: Kap. 4-5 und der dortige Anhang mit einem Abdruck von Beiträgen zur damaligen Diskussion um die Klassifikation von Eisen und Stahl.

20 Neben der chemischen Industrie (etwa Hounshell/Smith $1988 \mathrm{zu}$ DuPont) sind gut untersuchte Beispiele auch das 1900 gegründete Forschungslaboratorium von General Electric sowie die 1925 gegründeten Bell Laboratories (Birr 1957, Wise 1980, Reich 1985, Clayton/Algar 1989) und das 1920 bezogene Siemens'sche Forschungslabor (Trendelenburg 1975). Laut Cohen (1979: 35, 102, Anm. 83) hatten 1940 nur rund zehn Prozent aller US-Industrieunternehmen im Metallsektor ein materials department, 20 Jahre später hingegen schon mehr als die Hälfte. Zum Vergleich von (zögerlichen) Universitäten und MSE-naher Industrie siehe N.B. Hannay von den Bell Labs (Hannay in Roy, ed. (1970)) vs. George Krauss von der Lehigh University (Krauss in Roy, ed. (1970)).

21 Zum Kontext: Harwood in Roy, ed. (1970): 210, N.E. Promisel, Mitglied des Materials Advisory Board, National Academy of Sciences (in Ray in Roy, ed. (1970)) sowie BensaudeVincent 2001.

22 Auszüge aus einem solchen Vertrag bietet Harwood in Roy, ed. (1970: 8); im Anhang dieser Proceedings des National Colloquy on the Field of Materials befindet sich eine Übersicht aller universitärer Programme und wichtiger industrieller Forschungszentren im Bereich MSE mit Personenzahlangaben, Stand 1969.

23 Siehe Nielsen in Roy, ed. (1970): 17 zum Stand der Bezeichnungen von 85 Departments in USA und Kanada 1969 mit 25 Prozent Umbenennungen in Materials Science, Tendenz steigend. Vgl. Schwartz 1987, Groenewegen/Peters 2002, die die Abhängigkeit dieser Institutionalisierungsphasen von den Finanzierungsmodi unterstreichen.

24. Bensaude-Vincent (2001: 236) bietet eine Tabelle der Veränderung jener Anteile 1970 bis 1985 basierend auf Flemings 1999 und dem Jahrbuch der American Society for Metals (ASM).

25 Vgl. Cahn 2002: 3, 2003b: 468 über die zum Teil heftigen Debatten zur Umbenennung. In Pittsburgh wurde erst einige Jahre nach der Pensionierung des Metallurgen Robert Mehl das Department of Metallurgy and Materials Science zu Department of MSE. Vergleiche Bever (1988) und Flemings (2001: 854-857) zu analogen Entwicklungen am MIT, die 1972 bereits den Ausschlag zugunsten der Umbenennung in MSE gaben. Ab diesem Jahr gab es an der Northwestern University auch ein undergraduate program in MSE, für viele der entscheidende Indikator für die Existenz einer eigenständigen Disziplin.

26 Zur Geschichte jener Konzepte von Verhakungen und Versetzungen (dislocations) sowie von Fehlstellen beziehungsweise Dotierungen, deren Diffusion in Kristallgittern: Mott 1980: 125-177, Smith 1986b: 8-10, Weairde/Windsor 1987, Cahn 1995: 1520-1535, Seeger 2009.

27 Zu diesem Prozess Smith (1977: 3): „[P]rofessionalism in materials, until very recently, meant facilitating and cheapening the large-scale production“, ebd.: 27: „[A]doption in some measure of the physicist's view of the world without loss of an interest in practical diversity“.

28 Zitiert nach Cahn (2002: 3, 2003b: 468). Hollomon sah im Jahr 1958 das Geburtsjahr der ,materials science‘. (Vgl. Kranzberg/Smith 1979: 29.) Hollomon, der hier eine sonst eher bei Physikern zu findende Auffassung vertritt, war selbst am MIT in Metallurgie ausgebildeter Ingenieur und späterer Gründer der National Academy of Engineering in den USA.

29 Zitiert nach Cahn (2003b: 469), der diese Wortwahl ironisch als „inspired form of words“ kommentiert. Zur Geschichte dieser Gesellschaft siehe auch Jackson (1993: 8 f., 1993: 6987), wo in ein „nucleation stage“, eine „period of rapid growth"und eine Reifephase unterschieden wird.

30 Zu Denkstil und Sprache siehe Vogler 2008. 
31 So Melvin Calvin an Dr. Handler im Dezember 1973, zitiert im Vorwort in COSMAT 1974. Im Nachfolgebericht wird 1975 dann schon ausdrücklich der Singular verteidigt: "one should speak of materials science and engineering as an it rather than them" (COSMAT 1975: \$2,2). Eine Parallele bietet die Umbenennung der computer sciences in computer science, wie sie etwa von Ceruzzi (1989: 266 f.) beschrieben wird, wobei auch dort um 1959 noch der Zusatz „science(s) and engineering"mitgeschleift wurde, der später verschwand.

32 Zur Geschichte und Kritik dieses linearen Modells: Grandin 2004.

33 Zur Geschichte der Festkörperphysik und einiger Pioniere für MSE: Turnbull 1983: 2 f., Weaire/Windsor 1987, Weart 1988, Hoddeson 1977, Hoddeson u. a. 1992, Riordan/ Hoddeson 1997.

34 So etwa der Physiker Nevill F. Mott 1986 in seiner Autobiographie. In gewisser Weise ist das Stuttgarter Max-Planck-Institut für Festkörperforschung dieser Option gefolgt. Neben diesem gibt es noch das Max-Planck-Institut für Metallforschung (Köster 1949, 1961, Maier 2009), das als Kaiser-Wilhelm-Institut für Metallforschung 1921 in Neubabelsberg gegründet wurde, wo zuvor schon eine Zentralstelle für wissenschaftlich-technische Untersuchungen existiert hatte. 1922 wurde es nach Berlin-Dahlem verlegt und 1934 (nach seiner Schließung 1933 aufgrund Geldmangels) in Stuttgart neu gegründet.

35 Über Guertler siehe Anonym 1934; zur Zeitschrift für Metallkunde siehe das Editorial des Jubiläumsbandes vom umbenannten International Journal of Materials Research: 8.

36 Zur intensivierten Kooperation von Festkörperphysikern und Metallurgen seit Erscheinen der Acta Metallurgica siehe Cahn (1997: 163): „I still recall the tremendous intellectual impact created by the first volume“; vgl. Cahn (1995: 1556) zu dem von Paul Rosbaud (1896-1963) gegründeten Vorgängerorgan Progress in Metal Physics, unter anderen Zeitschriften beziehungsweise Buchreihen.

37 Über Tammanns Verwissenschaftlichung von metallurgisch wichtigen Prozessen wie Oxidation und Korrosion, Rekristallisation und Phasenumwandlungen, womit er und seine vielen Schüler in der ersten Hälfte des 20. Jahrhunderts großen Einfluss auf die Metallurgie in Deutschland ausübte, siehe Mehl 1948: 23 sowie Cahn (1995: 1540, $1554 \mathrm{f}$ ).

38 Zur Geschichte der MSE am MIT siehe Bever (1988) und Flemings (1999: 9-11). Übrigens wird metallurgy im angelsächsischen Sprachraum eingeschränkter nur in Bezug auf Praktiken der Metallherstellung benutzt. Diesen Hinweis verdanke ich Helmut Maier.

39 Über die einflußreiche Rolle von Köster, bis 1945 unter anderem Leiter des Forschungslabors der Vereinigten Stahlwerke, Herausgeber der Zeitschrift für Metallkunde und Mitglied im Reichsforschungsrat, ferner Professor für physikalische Metallurgie an der Stuttgarter Technischen Hochschule und Direktor des dortigen Kaiser-Wilhelm-Institut für Metallforschung, in der Organisation der Metallwirtschaft während des „Dritten Reiches“, siehe Maier 2007, 2009 und dort genannte weiterführende Quellen. Speziell zur Gründung der DGM, die aus einem Metallausschuß des Vereins Deutscher Ingenieure entstand, siehe Köster 1969.

40 Die vorstehenden Zahlen stammen aus Flemings (1999: 57) sowie Bensaude-Vincent (2001). Flemings Graphiken (2001: 857 f.) zeigen ab dem Jahr 1982 von 1700 auf knapp über 1000 Abschlüsse (2000) pro Jahr kontinuierlich fallende Tendenz, bis 1991 parallel zu den sinkenden Abschlüssen in chemical engineering, die danach aber wieder ansteigen, während MSE stagniert.

41 Wortgleich Flemings 1999: 17 und ähnlich 2001: 857. Über die zeitweise Blüte und den nachfolgenden Niedergang der Metallurgie als Leitwissenschaft der materials science siehe auch Cahn (2003b: 468) und dort zitierte weiterführende Texte.

42 Erste Anzeichen für eine solche bewußte Abgrenzung finden sich bereits in Roy (1970: viii, 11 - wiederholtes „we“-, 13): Abgrenzung gegenüber der „impatience of the metallurgist“, gegenüber der „occupational disease of the physicist [...] that he typically jumps into a field with a highy simplified model to describe a complex question".

43 Siehe über Polymerforschung und deren Geschichte für eine internalistische Sichtweise: Morawetz 1985, Seymour 1981, 1989, Carraher 1981, Furukawa 1998, Bijker 1995, Lemstra 2002: 48-51.

44 Dazu schreiben Kranzberg/Smith (1979: 29): „the sense of coherence is still only rudimentary [...] neither a metallurgist nor a polymer chemist nor a solid-state physicist working in the field of MSE tends to think of himself primarily as a materials scientist or engineer." 
45 Bensaude-Vincent 2001: 242, 2011. Bemerkenswerterweise ist „aggregation“ auch genau der Begriff, der von Calvin und anderen um 1974 benutzt wurde (vgl. hier Anm. 31); insofern trifft Bensaude-Vincent mit dieser Einschätzung eher den Stand der MSE von 1975. Für neuere Debatten vgl. ferner Dusastre 2002, 2003 und Bensaude-Vincent/ Hessenbruch 2004.

46 Als erste Annäherung an eine solche Liste des heutigen Kernfeldes jener Protagonisten nehme man die jeweils etwa hundert Namen umfassende Liste der Beitragenden zu den beiden - vom Stuttgarter Max-Planck-Institut für Metallforschung koordinierten European White Books, siehe Rühle 2002, Dosch/van de Voorde 2009.

47 Siehe Allen/Thomas (1998: vi) zu dem dort erhobenen und meines Erachtens auch eingelösten Anspruch einer generischen Strukturbeschreibung quer durch die Stoffklassen („materials catholic“). Die Möglichkeit einer „alle Werkstoffgruppen erfassenden, einenden und zugleich ordnenden Betrachtungsweise" wurde schon von Schatt (1978: 361) erkannt, der sie damit begründete, „warum es nur eine Werkstoffwissenschaft gibt“ (Hervorhebung im Original), aber in seinem Lehrbuch (1970) nicht eingelöst; vgl. ferner Ashby und Jones 1980.

48 Der so nicht im OED auftauchende Begriff findet sich als strategisch zur Abgrenzung eingesetzter Terminus zum ersten Mal bei Turnbull (1983: 1); für neuere Verwendungen siehe etwa Rühle (2002: 37), dort zusammen mit der ebenfalls ungewöhnlichen Solid State Chemistry, wobei MSE in der Mitte zwischen den Extremen von exploratory research der Chemie und Physik einerseits und der Nähe zum final product der material technology andererseits angesiedelt wird.

49 Siehe zu diesen Disziplinengenesen etwa Meinel (1985, 1987) Stichweh (1984, 1992, 1993), Servos 1990; zum Kontrast zwischen dieser typischen Disziplinentwicklung und der der MSE: Kranzberg/Smith (1979: 30). Explizite Vergleiche zur Genese der physikalischen Chemie mit lange andauernden Konflikten zwischen Physikern und Chemikern sowie zur Geologie finden sich bei Cahn (1997: 160 f., 2001b: passim). Alternative Modelle diskutieren u.a. Ziman 1996, Shinn 2002 und dort jeweils genannte weiterführende Texte.

50 Aussagekräftig ist das Editorial in Nature Materials 2003: „[O]ther academics departments, notably physics, chemistry, and chemical and mechanical engineering, are also facing waning undergraduate enrolment. Because these departments increasingly tend to view materials science and engineering as a subset of their own field, they all end up competing for the same students, thereby threatening their own existence."(Dusastre 2003).

\section{Literatur}

Aldinger, Fritz, 1999. Werkstoffe, die die Welt verändern - Hochleistungskeramiken machen den etablierten Materialien Konkurrenz. Physikalische Blätter, 55, Nr. 11, 31-37.

Aldinger, Fritz/Baumard, Jean-François, 2002. Advanced Ceramic Materials: Summary of Possible Applications. In: Manfred Rühle u. a., Hg., 2002. European White Book on Fundamental Research in Materials Science, Stuttgart: Max-Planck-Institut für Metallforschung, 26-31.

Allen, Samuel M./Thomas, Edwin L., 1998. The Structure of Materials, New York: Wiley.

Amstutz, Eduard, 1955. EMPA: Rückblick und Ausblick, Schweizer Archiv für angewandte Wissenschaft \& Technik, 21, 347-359.

Anonym., 1930. Entwicklung der nichtpreußischen Materialprüfungsanstalten, Mitteilungen der deutschen Materialprüfungsanstalten, Heft 6/8, 81-98.

Anonym., 1934. Ein neues Ordinariat für Metallkunde, Metallwirtschaft, 13, 25-26.

Ashby, Michael F./Jones, David R.H., 1980. Engineering Materials, Oxford: Pergamon Press; in dt. Übers. v. P.P. Schepp: Ingenieurwerkstoffe. Einführung in ihre Eigenschaften und Anwendungen, Berlin: Springer, 1986.

Bach, Carl/Baumann, Richard, [1914] 1921. Festigkeitseigenschaften und Gefügebilder der Konstruktionsmaterialien, 2. stark erw. Aufl. Berlin: Springer. 
Baumann, Richard, 1912. Das Materialprüfungswesen und die Erweiterung der Erkenntnisse auf dem Gebiete der Elastizität und Festigkeit in Deutschland während der letzten 4 Jahrzehnte, Beiträge zur Geschichte der Geschichte der Technik und Industrie 4, 147-195.

Baumann, Richard, 1920. Metallographie. In: Otto Lueger, Hg., Lexikon der gesamten Technik und ihrer Hilfswissenschaften, Stuttgart/Leipzig: Dt. Verlags-Anstalt, Ergänzungs-Bd. 1, 449-454.

Bauschinger, Johann, 1879. Ueber Einrichtung und Ziele von Prüfunganstalten für Baumaterialien und die Classification der letzteren, insbesondere des Eisens und Stahls, Zeitschrift des Vereines deutscher Ingenieure, 23, 49-66 und Tafelblätter 4-6.

Bensaude-Vincent, Bernadette, 2001. The Construction of a Discipline: Materials Science in the United States. Historical Studies in the Physical Sciences, 31, 223-248.

Bensaude-Vincent, Bernadette, 2011. The Concept of Materials in Historical Perspective. NTM. Zeitschrift für Geschichte der Wissenschaften, Technik und Medizin, 19, XX-XX.

Bensaude-Vincent, Bernadette/Hessenbruch, Arne, 2004. Materials Science: A Field about to Explode? Nature Materials, 3, 345-347.

Bever, Michael B., 1988. Metallurgy and Materials Science and Engineering at MIT: 1865-1988, Cambridge, MA: MIT Press.

Bijker, Wiebe E., 1995. Of Bicycles, Bakelites, and Bulbs. Towards a Theory of Sociotechnical Change, Cambridge, MA: MIT Press.

Binnig, Gerd/Rohrer, Heinrich, 1985. The Scanning Tunneling Microscope. Scientific American, 253, 50-56.

Binnig, Gerd/Rohrer, Heinrich 1987. The Scanning Tunneling Microscope - From Birth to Adolescence. Review of Modern Physics, 59, 615-625.

Birr, Kendall, 1957. Pioneering in Industrial Research: The Story of the General Electric Research Laboratory, Washington, D.C.

Blind, Dieter/Werner, Gerhard, 1984. 100 Jahre Materialprüfung in Stuttgart, Wechselwirkungen, 65-75.

Bragg, Lawrence, 1945. The History of X-Ray-Analysis, London: Longmans Green \& Co.

Braun, Hans-Joachim/Herla, Alexandre, Hg., 2002. Materials: Research, Development and Applications, Turnbout: Brepols.

Cahn, Robert W., 1995. Physics of Materials. In: Laurie M. Brown, Abraham Pais und Brian Pippard, Hg. Twentieth-Century Physics, Bristol: Institute of Physics Publications, Bd. 3: 15051564 .

Cahn, Robert W., 1997. History of Physical Metallurgy and of Materials Science, Acta Metallurgica Sinica 33, 2 (197): 157-164.

Cahn, Robert W., 2001a. The Coming of Materials Science, Oxford: Pergamon.

Cahn, Robert W., 2001b. Genesis by Definition. Nature, 410, 307.

Cahn, Robert W., 2002. The Science of Dirt. Nature Materials, 1, 3-4.

Cahn, Robert W., 2003a. Materials Departments Are Facing Up to Education and Recruitment Concerns. Nature Materials, 2, 128-129.

Cahn, Robert W., 2003b. Has the Child of Metallurgy Walked Out on Its Parent? MRS Bulletin, Juli, 468-469.

Canby, Thomas Y., 1989. Advanced Materials - Reshaping Lives. National Geographic, 176, 746781.

Carraher, Charles E.,1981. History of Polymer Education-USA. In: Raymond B. Seymour, Hg., History of Polymer Science and Technology, New York: Dekker 1981: 1237-1261.

Ceruzzi, Paul, 1989. Electronics, Technology and Computer Science, 1940-1975. A Coevolution, Institute of Electrical and Electronics Engineers Annals of the History of Computing, 10, 4, 257 275.

Charles, James A./Greer, Alan Lindsay. 2005. Light Blue Materials. The Department of Materials Science and Metallurgy, University of Cambridge - A History. Cambridge.

Cohen, Morris, Hg., 1979. Materials Science and Engineering: Its Evolution, Practice and Prospects, Journal of Materials Education 1, 1-102.

Chubin, David E., 1976. The Conceptualization of Scientific Specialties. Sociological Quarterly, 17, 448-476.

Clayton, Robert J./Algar, Joan, 1989. General Electric Company Research Laboratory 1919-84, New York: IEE.

Cockayne, David, 2002. Electron Microscopy. Better Vision with Electron Lenses. Nature Materials, 1, 7-8. 
COSMAT 1974. Materials and Man's Needs: Materials Science and Engineering, Washington, D.C. Summary Report by the Committee on the Survey of Materials Science and Engineering. [http://books.nap.edu/catalog.php?record_id=10435, letzter Zugriff: 26. September 2010].

COSMAT 1975. Materials and Man's Needs: Materials Science and Engineering. Bd. 1: The History, Scope and Nature of Materials Science and Engineering. Bd. 2: The Needs, Priorities, and Opportunities for Materials Research. Bd. 3: The Institutional Framework for Materials Science. Bd. 4: Aspects of Materials Technology Abroad. Supplementary Report of the Committee on the Survey of Materials Science and Engineering. Washington, D.C.: National Academy of Sciences.

COSMAT 1989. Materials Science and Engineering for the 1990's. Report by the Committee on Materials Science and Engineering, National Research Council, Washington, D.C.

Cunliffe, Barry, Hg., 1994. The Oxford Illustrated Prehistory of Europe. Oxford: Oxford University Press.

Day, Peter, 1995. Michael Faraday as a Materials Scientist, Materials World, 3, 374-376.

Deutsche Gesellschaft für Materialkunde, Hg. 2004. Zur Geschichte der DGM im Spiegel der Zeitschrift für Metallkunde. Oberursel: Deutsche Gesellschaft für Materialkunde.

Ditchen, Henryk, 2009. Die Beteiligung Stuttgarter Ingenieure an der Planung und Realisierung der Reichsautobahn unter besonderer Berücksichtigung des Netzwerks von Fritz Leonhardt und Otto Graf, Berlin: Logos.

Domke, Wilhelm, [1964] 1999. Werkstoffkunde und Werkstoffprüfung, 10. Auf., Essen: Girardet.

Dosch, Helmut/Van de Voorde, Marcel H.,Hg., 2009. GENNESYS White Paper. A New European Partnership between Nanomaterials Science E Nanotechnology and Synchrotron Radiation and Neutron Facilities. Stuttgart: Max-Planck-Institut für Metallforschung.

Dresselhaus, Mildred Spiewak, 1992. Materials Science and Cultural Change, New Literary History, 23, 839-844.

Dusastre, Vincent 2002. Editorial: Our Changing Nature, Nature Materials, 1: 1.

Dusastre, Vincent 2003. Editorial: A Matter of Survival, Nature Materials, 2: 1.

Dusastre, Vincent 2009. Editorial. at the Beginning of Volume 100, International Journal of Materials Research, 100, 1: 2-8.

Eckert, Michael, 1993. Die Atomphysiker. Eine Geschichte der theoretischen Physik am Beispiel der Sommerfeldschule, Braunschweig: Vieweg.

Edyvean, Robert G. J./Hammond, C., 1997. The Metallurgical Work of Henry Clifton Sorby and an Annotated Catalogue of His Extant Metallurgical Samples. Historical Metallurgy 31, 2, 5485.

Ewald, Paul Peter, 1962. Fifty Years of X-Ray Diffraction, Utrecht: Oesthoek.

Fellman, Megan, 2005. World's First Materials Science and Engineering Department Turns 50 Years Old, Northwestern University Observer Online, 20. Oktober 2005. [http://www. northwestern.edu/observer/issues/2005/10/20/materials.html, letzter Zugriff am 20. Dezember 2010]

Flemings, Merton C., 1999. What Next for Departments of Materials Science and Engineering. Annual Review of Materials Science, 29, 1-24.

Flemings, Merton C., 2001. Why Materials Science and Engineering Is Good for Metallurgy. Metallurgical and Materials Transactions, 32 A, 853-860.

Flemings, Merton C./Cahn, Robert W., 2000. Organization and Trends in Materials Science and Engineering Education in the US and Europe. Acta Materialia, 48, 371-383.

Föppl, Ludwig/Wintergerst, S./Rüsch, H 1959. Die Materialprüfung an der Technischen Hochschule München, Materialprüfung, 1, 142-146

Furukawa, Yasu, 1998. Inventing Polymer Science: Staudinger, Carothers and the Emergence of Macromolecular Chemistry, Philadelphia: University of Pennsylvania Press.

Geison, Gerald L./Creager, Angela N.K., 1999. Introduction: Research Materials and Model Organisms in the Biological and Biomedical Sciences. Studies in the History and Philosophy of the Biological and Biomedical Sciences, 30, 315-318.

Gieryn, Thomas F., 1983. Boundary-work and the Demarcation of Science from Non-science. American Sociological Review, 48, 781-795.

Gieryn, Thomas F., 1995. Boundaries of Science. In: Jasanoff, Sheila, Hg., Handbook of Social and Technology Studies, London/New Delhi: Society for Social Studies of Science, 398-403.

Grandin, Karl, Hg., 2004. The Science Industry-Nexus: History, Polic. Sagamore Beach, Mass.: Science History Publications Implications [= Nobel Symposium 123, 2002]. 
Granek, Galina/Hon, Giora, 2008. Search for Asses, Finding a Kingdom: The Story of the Invention of the Scanning Tunnelling Microscope (STM). Annals of Science, 65, 101-125.

Groenewegen, Peter/Peters, Lois, 2002. The Emergence and Change of Materials Science and Engineering in the United States. Science, Technology and Human Values, 27, 111-133.

Hacking, Ian, 2002. Historical Ontology, Cambridge, MA: Harvard University Press [Historische Ontologie, Zürich: Chronos, 2005].

Haka, Andreas, 2011. Flügel aus „Schwarzem Gold“. Zur Geschichte der Faserverbundwerkstoffe. NTM. Zeitschrift für Geschichte der Wissenschaften, Technik und Medizin, 19, XX-XX..

Hammond, C., 1989. The Contribution of Henry Clifton Sorby to the Study of Reflected Light Microscopy of Iron and Steel. Historical Metallurgy, 23, 1, 1-8.

Hanemann, Heinrich/Schrader, Angelika, Hg., 1933-1952. Atlas Metallographicus, Berlin: Bornträger.

Heizer, Robert F., 1962. The Background of Thomsen's Three-Age System, Technology and Culture 3, 259-266.

Hennig, Jochen, 2004. Changes in the Design of Scanning Tunneling Microscopic Images from 1980 to 1990 . Techné, 8, 1-20.

Hennig, Jochen, 2011. Bildpraxis. Visuelle Strategien in der frühen Nanotechnologie, Bielefeld: transkript.

Hentschel, Klaus, 2002. Mapping the Spectrum. Techniques of Visual Representation in Research and Teaching, Oxford: Oxford University Press.

Higham, Norman, 1963. A Very Scientific Gentleman: The Major Achievements of Henry Clifton Sorby, Oxford: Pergamon Press.

Hoddeson, Lillian, 1977. The Roots of Solid-state Research at Bell Labs, Physics Today, März, 23-30.

Hoddeson, Lillian/Braun, Ernest/Teichmann, Jürgen/Weart, Spencer, 1992. Out of the Crystal Maze. Chapters from the History of Solid-State Physics, Oxford: Oxford University Press.

Hounshell, David/Smith, John K., 1988. Science E Corporate Strategy, DuPont R E D, 1902-1980, Cambridge, MA.

Hummel, Rolf, [1998] 2004. Understanding Materials Science: History, Properties, Applications. 2. erweiterte Aufl. Berlin: Springer.

Hunt, Bruce, 1997. Doing Science in a Global Empire: Cable Telegraphy and Electrical Physics in Victorian Britain. In: Bernard Lightman, Hg., Victorian Science in Context, Chicago: Chicago University Press: 312-353.

Jackson, Kenneth/Roy, Rustum/Voorhoeve, R.J.H./Picraux, Thomas, Hg., 1993. MRS $20^{\text {th }}$ Anniversary, Materials Research Society Bulletin, 18, 69-87.

Johnson, Ann, 2009. Material Experiments: Environment and Engineering Institutions in the Early American Republic, Osiris, 24, 53-74.

Kahlow, Andreas, 1991. Materials in $19^{\text {th }}$ Century Germany, History and Technology, 7, 255-266.

Kingery, W. David, 1986. High-Technology Ceramics: Past, Present and Future, Westerville, Ohio: American Ceramics Society.

Klein, Ursula/Lefèvre, Wolfgang, 2007. Materials in $18^{\text {th }}$-Century Science. A Historical Ontology, Cambridge, MA: MIT Press.

Kloos, K.H./Berger, Christina, 2007. 100 Jahre staatliche Materialprüfanstalt Darmstadt und 80 Jahre Teilgebiet und Institut für Werkstoffkunde an der TH Darmstadt, Materialwissenschaft und Werkstofftechnik, 38, 310-346.

Köster, Werner, 1949. 25 Jahre Kaiser-Wilhelm-Institut für Metallforschung 1921-1946, Stuttgart: Riederer.

Köster, Werner, 1961. Max-Planck-Institut für Metallforschung in Stuttgart, Jahrbuch der MaxPlanck-Gesellschaft, Teil II: 600-626.

Köster, Werner, 1969. 50 Jahre Deutsche Gesellschaft für Metallkunde, Metall 23, 661-666.

Krankenhagen, Gernot/Laube, Horst, 1983. Werkstoffprüfung. Von Explosionen, Brüchen und Prüfungen, Reinbek bei Hamburg: Rowohlt.

Kranzberg, Melvin/Smith, Cyril Stanley, 1979. Materials in History and Society. Materials Science and Engineering, 37, 1-39.

Kunckel, Johannes, [1679] 1972. Ars Vitraria Experimentalis, oder Vollkommene Glasmacherkunst. Frankfurt, Reprint hg. Günther Stein, Hildesheim: Ohms.

Laitko, Hubert, 2002. Die Disziplin als Strukturprinzip und Entwicklungsform der Wissenschaft. In: Ekkehard Höxtermann und Uwe Hoßfeld, Hg., Die Entstehung biologischer Disziplinen, Berlin: Verlag für Wissen und Bildung: 19-55. 
Lemaine, Gérard/MacLeod, Roy/Mulkay, Michael/Weingart, Peter, Hg., 1976. Perspectives on the Emergence of Scientific Disciplines, Mouton/Paris: Maison des Sciences de l'Homme.

Lemmerich, Jost, 1996. 100 Jahre Röntgenstrahlen. Ausstellung, Universität Würzburg.

Lemstra, Pieter J., 2002. Soft Materials and Polymers: The Rise and Decline of Polymer Science \& Technology in Europe. In: Manfred Rühle, Helmut Dosch, Eric J. Mittemeijer und Marcel H. Van de Voorde, Hg., 2002. European White Book on Fundamental Research in Materials Science, Stuttgart: Max-Planck-Institut für Metallforschung: 48-50.

Leon, Alfons, 1912. Die Entwicklung und die Bestrebungen der Materialprüfung. Wien: Verein des österreichischen Verbandes für die Materialprüfungen der Technik.

Luxbacher, Günther, 2010. Werkstoff, Sparstoff, Heimstoff. Metallforschung in Deutschland 1920-1970, in: Karin Orth und Willi Oberkrome, Hg, 2010. Die Deutsche Forschungsgemeinschaft 1920-1970, Stuttgart: Steiner, 163-181.

Luxbacher, Günther, 2011. „Für bestimmte Anwendungsgebiete best geeignete Werkstoffe...finden“. Zur Praxis der Forschung an Ersatzstoffen für Metalle in den deutschen AutarkiePhasen des 20. Jahrhunderts. NTM. Zeitschrift für Geschichte der Wissenschaften, Technik und Medizin, 19, XX-XX.

Mämpel, Uwe, 1985. Keramik. Von der Handform zum Industrieguß. Reinbek: Rowohlt.

Maier, Helmut, 2000. Materials Science. In: Arne Hessenbruch, Hg., Reader's Guide to the History of Science, London/Chicago: Fitzroy Dearborn, 442-444.

Maier, Helmut, 2002. Ideologie, Rüstung und Ressourcen. Das Kaiser-Wilhelm-Institut für Metallforschung und die „deutschen Metalle“ 1933-1945, in: Ders., Hg., Rüstungsforschung im Nationalsozialismus: Organisation, Mobilisierung und Entgrenzung der Technikwissenschaften, Göttingen: Wallstein, 2002, 357-388.

Maier, Helmut, 2006. Leichter als Krupp-Stahl? Zur ,material culture' des Aluminiums seit der Mitte des 19. Jahrhunderts. In: Torsten Meyer/Marcus Popplow, Hg., Technik, Arbeit und Umwelt in der Geschichte, New York: Waxmann, 2006, 265-279.

Maier, Helmut, 2007. Forschung als Waffe. Rüstungsforschung in der Kaiser-Wilhelm-Gesellschaft und das Kaiser-Wilhelm-Institut für Metallforschung 1900 bis 1945/48, Göttingen: Wallstein.

Maier, Helmut, 2009. Ideology, Armament and Resources: The KWI for Metal Research and the ,German Metals‘ 1933-1945. In: Susanne Heim, Carola Sachse und Mark Walker, Hg., The Kaiser-Wilhelm Society under National Socialism, New York: Cambridge University Press, 2009, 253-282.

Martens, Adolf, 1880. Das mikroskopische Gefüge und die Kristallisationen des Roheisens, Zeitschrift des Vereines Deutscher Ingenieure, 24: 397-406 und Tafeln XX-XXI.

Martens, Adolf, 1898. Handbuch der Materialkunde für den Maschinenbau, Berlin: Springer.

Martens, Adolf/Guth, Max, 1904. Das königliche Materialprüfungsamt der Technischen Hochschule Berlin, Berlin: Springer.

Mehl, Robert Franklin, 1948. A Brief History of the Science of Metals, New York: American Institute of Mining and Metallurgical Engineers.

Meinel, Christoph, 1985. Reine und angewandte Chemie - Die Entstehung einer neuen Wissenschafts-konzeption in der Chemie der Aufklärung. Berichte zur Wissenschaftsgeschichte, 8, 25-45.

Meinel, Christoph, 1987. Zur Sozialgeschichte des chemischen Hochschulfaches im 18. Jahrhundert. Berichte zur Wissenschaftsgeschichte, 10, 147-168.

Mody, Cyrus, 2006. Corporations, Universities and Instrumental Communities. Commercializing Probe Microscopy, 1981-1996, Technology and Culture, 47: 56-80.

Morawetz, Herbert, 1985. Polymers: The Origins and Growth of a Science, New York: Wiley.

Mott, Nevill Francis, Hg., 1980. The Beginnings of Solid State Physics, Proceedings of the Royal Society London, A 371, 1-177.

Mott, Nevill Francis, 1986. A Life in Science, London: Taylor \& Francis.

Newbury, Dale E./Williams, David B., 2000. The Electron Microscope: The Materials Characterization Tool of the Millenium, Acta Materialia 48, 323-346.

Nüßlein, Rudolf, 1965. Die Geschichte der ersten deutschen Universalprüfmaschine, Materialprüfung, 7, 425-432.

Opitz, Herbert/Dude, Werner, [1964] 1977. Allgemeine Werkstoffprüfung für Ingenieurschulen. 7. Aufl., Leipzig: VEB Fachbuchverlag.

Piersig, Wolfgang, 2007. Adolf Martens - Erinnerungen an den Nestor - Materialprüfungen der Technik, Norderstedt: GRIN-Verlag. 
Piersig, Wolfgang, 2008. Emil Heyn. Nestor der Technikwissenschaften - Metallkunde und Metallographie, Norderstedt: GRIN-Verlag.

Preuß, Ernst, [1913] 1927. Die praktische Nutzanwendung der Prüfung des Eisens durch Ätzverfahren mithilfe des Mikroskops, 3. Aufl., Berlin: Springer.

Psaras, Peter A./Langford, H. Dale, Hg., 1987. Advancing Materials Research. Washington: National Academy Press.

Pusch, Richard, 1979. Die Geschichte der Metallographie mit besonderer Berücksichtigung der mikroskopischen Prüfverfahren. Praktische Metallographie, 16: 26-35, 79-87, 294-300, in mehreren Teilfolgen, gesammelt in: Ders., Die Geschichte der Metallographie, unter Berücksichtigung der mikroskopischen Prüfverfahren. Düsseldorf: Verein Deutscher Eisenhüttenleute 1976 [Fachausschussbericht 9005].

Queisser, Hans, 1985. Kristallene Krisen. Mikroelektronik, München. Piper.

Qing, Lin, 1995. Zur Frühgeschichte des Elektronenmikroskops, Stuttgart: GNT-Verlag.

Radkau, Joachim, 2007. Holz. Wie ein Naturstoff Geschichte schreibt, München: Oekom.

Ramsauer, Carl, 1943. Elektronenmikroskopie: Bericht über Arbeiten des AEG Forschungsinstituts 1930-1942, 3. erw. Aufl., Berlin: Springer.

Rasmussen, Nicholas, 1997. Picture Control: The Electron Microscope and the Transformation of Biology in America, 1940-1960, Stanford: Stanford University Press.

Raymond, Ronald, 1986. Out of the Fiery Furnace - The Impact of Metal on the History of Mankind. University Park, PA: Pennsylvania State University Press.

Réaumur, René-Antoine F. de, 1722. L'art de convertir le fer forgé en acier, Paris: Brunet.

Reich, Leonard, 1985. The Making of American Industrial Research. Science and Business at GE and Bell, 1876-1926, New York: Cambridge University Press.

Reinhardt, Carsten, 2006. Shifting and Rearranging. Physical Methods and the Transformation of Modern Chemistry. Sagamore Beach, MA: Science History Publications.

Reinhardt, Carsten, 2011. Habitus, Hierarchien und Methoden: „Feine Unterschiede“ zwischen Physik und Chemie. NTM. Zeitschrift für Geschichte der Wissenschaften, Technik und Medizin, 19, im Druck.

Riordan, Michael/Hoddeson, Lillian, 1997. Crystal Fire. The Birth of the Information Age, New York: W.W. Norton.

Roy, Rustum, Hg., 1970. Materials Science and Engineering in the United States, University Park, PA: Pennsylvania State University Press.

Rühle, Manfred/Helmut Dosch/Eric J. Mittemeijer/Marcel H. Van de Voorde, Hg., 2002. European White Book on Fundamental Research in Materials Science, Stuttgart: Max-Planck-Institut für Metallforschung.

Ruske, Walter, 1971. 100 Jahre Materialprüfung in Berlin, Berlin: Bundesanstalt für Materialprüfung 1971.

Ruske, Walter/Becker, Gerhard W./Czichos, Horst, 1996. 125 Jahre Forschung und Entwicklung, Prüfung, Analyse, Zulassung, Beratung und Information in Chemie- und Materialtechnik, Berlin: Bundesanstalt für Materialforschung und -prüfung.

Sass, Stephen L., 1998. The Substance of Civilization. Materials and Human History from the Stone Age to the Age of Silicon, New York: Arcade.

Schatt, Werner, [1972] 2003. Einführung in die Werkstoffwissenschaft, Leipzig: Deutscher Verlag für Grundstoffindustrie. 9. Aufl. Weinheim: Wiley-VCH.

Schatt, Werner, 1978. Wesen und Bestimmung der Werkstoffwissenschaft, Neue Hütte, 23, 360-362.

Scheel, Bernd, 1989. Egyptian Metalworking and Tools, Aylesbury: Shire.

Schulz, Ernst Hermann, 1949. 100 Jahre Werkstoffprüfung, Zeitschrift des Vereins Deutscher Ingenieure, 91, 141-147.

Schummer, Joachim, 2011. Nanotechnologie: Eine neue Dynamik an der Schnittstelle zwischen Wissenschaft und Öffentlichkeit. NTM. Zeitschrift für Geschichte der Wissenschaften, Technik und Medizin, 19, im Druck.

Schwartz, Lyle H. 1987. Materials Research Laboratories: Reviewing the First Twenty-five Years. In: Peter A. Psaras und H. Dale Langford, Hg. Advancing Materials Research. Washington: National Academy Press: 35-48.

Seeger, Alfred, 2009. Verhakungen, Dislocations, Solitons, and Kinks. International Journal of Materials Research, 100, 24-36.

Servos, John W., 1990. Physical Chemistry from Ostwald to Pauling. The Making of a Science in America, Princeton: Princeton University Press. 
Seymour, Raymond B., Hg., 1981. History of Polymer Science and Technology, New York: Dekker [= Sonderheft 6/7 des Journal of Macromolecular Science A15, 1980].

Seymour, Raymond B., Hg., 1989. Pioneers in Polymer Science, Dordrecht: Kluwer.

Shinn, Terry/Joerges, Bernward, 2001. Instrumentation between Science, State and Industry. Dordrecht: Kluwer [Sociology of Science Yearbook, 22].

Shinn, Terry, 2002. The Triple Helix and the New Production of Knowledge. Pre-packaged Thinking on Science and Technology, Social Studies of Science, 32: 599-614.

Shumway, David/Messer-Davidow, Ellen, 1991. Disciplinarity: An Introduction. Poetics Today, 12, 2: 201-225.

Sigwart, Hans, 1986. Aus der Geschichte der Werkstoffprüfung und der Festigkeitslehre, Berlin: Deutscher Verband für Materialprüfung.

Smith, Cyril Stanley, [1960] 1988. A History of Metallography: The Development of Ideas on the Structure of Metals before 1890, Chicago: University of Chicago Press [Reprint Cambridge, MA: MIT Press].

Smith, Cyril Stanley, 1961. Retrospective Notes on a Changing Profession, Archaeomaterials 1, $3: 11$.

Smith, Cyril Stanley, Hg, 1965. The Sorby Centennial Symposium on the History of Metallurgy, New York: Gordon \& Breach.

Smith, Cyril Stanley, Hg., 1968. Sources for the History of the Science of Steel, 1532-1786, Cambridge, MA: MIT Press.

Smith, Cyril Stanley, 1969. The Development of Ideas on the Structure of Metals. In: Marshall Clagett, Hg., Critical Problems in the History of Science. Madison, WI: 467-498.

Smith, Cyril Stanley, 1977. Metallurgy as a Human Experience. Materials Park, Ohio: American Society for Metals.

Smith, Cyril Stanley, 1986a. On Material Structure and Human History, Annual Review of Materials Science, 16, 1:11.

Smith, Cyril Stanley, 1986b. Retrospective Notes on a Changing Profession, Archaeomaterials, 1, 3-11

Sonnemann, Rolf/Friedrich, Eckhart, 1978. Die Werkstoffwissenschaft im Spiegel der Geschichte der Technischen Universität Dresden, Neue Hütte, 23, 357-360.

Sonnenberg, Gerhard Siegfried, 1968. 100 Jahre Sicherheit. Beiträge zur technischen und administrativen Entwicklung des Dampfkesselwesens in Deutschland, Düsseldorf: Verein Deutscher Ingenieure.

Sorby, Henry Clifton, 1897. Fifty Years of Scientific Research. An Address Delivered before the Members of the Sheffield Literary and Philosophical Society [am 2. Febr. 1897], Sheffield: Independent Press.

Stichweh, Rudolf, 1984. Zur Entstehung der wissenschaftlichen Disziplinen: Physik in Deutschland, 1740-1890, Frankfurt a. M.: Suhrkamp.

Stichweh, Rudolf, 1992. The Sociology of Scientific Disciplines. Science in Context, 5, 3-15.

Stichweh, Rudolf, 1993. Wissenschaftliche Disziplinen - Bedingungen ihrer Stabilität im 19. und 20. Jahrhundert. In: Jürgen Schriewer, Edwin Keiner und Christophe Charle, Hg., Sozialer Raum und akademische Kulturen, Frankfurt a. M.: Peter Lang: 235-250.

Strahm, Christian, 1995. Die Anfänge der Metallurgie in Mitteleuropa. Helvetia Archaeologica, 25, Nr. 97: 2-39.

Stratmann, Martin, 2006. Materials Science: A Cross-cutting Area between Scientific Research and Industrial Development. Max Planck Forum, 7, Mai: 209-227.

Thiel, Christian/Peckhaus, Volker, Hg., 1999. Disziplinen im Kontext, München: Fink.

Timoshenko, Stephen P., 1953. History of the Strength of Materials, New York: McGraw Hill.

Trendelenburg, Ferdinand, 1975. Aus der Geschichte der Forschung im Hause Siemens, Düsseldorf: Verein Deutscher Ingenieure [= Technikgeschichte in Einzeldarstellungen, 31].

Turnbull, David, 1983. A Commentary on the Emergence and Evolution of ,Materials Science'. Annual Review of Materials Science, 13, 1-7.

Tylecote, Ronald Frank, [1976] 1992. A History of Metallurgy, 2. Aufl., London: The Institute of Materials.

Ude, Hans, 1935. Zur Geschichte der Eisenbahnwerkstoffe, Technikgeschichte 24: 38-60.

Urban, Klaus, 1972. Die Ziel-Inhalt-Methode-Relation in der Lehre der Werkstoffwissenschaft für Ingenieure des Maschinenbaus an Hochschulen der DDR. TU Dresden: Dissertation A.

Vogler, Daniela, 2008. Der technikwissenschaftliche Denkstil in seiner sprachlichen Manifestation, dargestellt am Beispiel der Werkstoffwissenschaft, Hamburg: Kovač. 
Wagner, Donald B., 2000. Metallurgy. In: Arne Hessenbruch, Hg., Reader's Guide to the History of Science, London/Chicago: Fitzroy Dearborn: 472-473.

Weaire, Denis J./Windsor, Colin G., Hg, 1987. Solid State Science. Past, Present and Predicted, Bristol: Hilger.

Weart, Spencer, 1988. The Birth of the Solid-state Physics Community, Physics Today, Juli, 38-45.

Werner, Otto, 1964. Adolf Martens - zur 50. Wiederkehr seines Todestages, Materialprüfung, 6, 249-250.

Wise, George, 1980. A New Role for Professional Scientists in Industry: Industrial Research at General Electric 1909-16. Technology and Culture, 21, 408-429.

Wickramasinghe, H. Kumar, 2000. Progress in Scanning Probe Microscopy, Acta Materialia, 48, 347-358.

Wöhler, August, 1877. Denkschrift über die Einführung einer staatlich anerkannten Classification von Eisen und Stahl, Zeitschrift des Vereines deutscher Ingenieure 21, 518-523.

Ziman, John, 1995. Some Reflections on Physics as a Social Institution. In: Laurie M. Brown, Abraham Pais und Brian Pippard, Hg., Twentieth-Century Physics, Bd. 3: Bristol: Institute of Physics Publications, 2041-2059.

Ziman, John, 1996. Post-Academic Science: Constitution Knowledge with Networks and Norms, Nature, 382, 751-754.

Zweckbronner, Gerhard, 1985. Materialprüfung in Wechselwirkung zur technisch-industriellen Entwicklung, dargestellt am Beispiel Carl Bach, Ferrum, 56, 39-42.

Klaus Hentschel

Abteilung für Geschichte der

Naturwissenschaften und Technik

Historisches Institut der Universität Stuttgart

Keplerstr. 17

70174 Stuttgart

Germany

E-Mail: klaus.hentschel@po.hi.uni-stuttgart.de

URL: www.uni-stuttgart.de/hi/gnt/hentschel 